\title{
Scattering amplitude and bosonization duality in general Chern-Simons vector models
}

\author{
Shuichi Yokoyama \\ Yukawa Institute for Theoretical Physics, Kyoto University, \\ Sakyo, Kyoto 606-8502, Japan \\ Research and Education Center for Natural Sciences, Keio University, \\ Hiyoshi, Kanagawa 223-8521, Japan \\ E-mail: shuichi.yokoyama@yukawa.kyoto-u.ac.jp
}

ABSTRACT: We present the exact large $N$ calculus of four point functions in general ChernSimons bosonic and fermionic vector models. Applying the LSZ formula to the four point function we determine the two body scattering amplitudes in these theories taking a special care for a non-analytic term to achieve unitarity in the singlet channel. We show that the S-matrix enjoys the bosonization duality, an unusual crossing relation and a non-relativistic reduction to Aharonov-Bohm scattering. We also argue that the S-matrix develops a pole in a certain range of coupling constants, which disappears in the range where the theory reduces to the Chern-Simons theory interacting with free fermions.

Keywords: 1/N Expansion, Chern-Simons Theories, Duality in Gauge Field Theories, Scattering Amplitudes

ArXIV EPRINT: 1604.01897 


\section{Contents}

1 Introduction 1

2 General Chern-Simons fermion vector model 2

$\begin{array}{lll}3 & \text { Four point function of fermions } & 4\end{array}$

4 S-matrix and duality $\quad 10$

$\begin{array}{lll}4.1 & \text { T-channel } & 12\end{array}$

$\begin{array}{lll}4.2 & \text { S-channel } & 14\end{array}$

$\begin{array}{llr}5 & \text { Discussion } & 17\end{array}$

$\begin{array}{ll}\text { A Regularization } & 19\end{array}$

$\begin{array}{ll}\text { B Construction of asymptotic states } & 20\end{array}$

$\begin{array}{ll}\text { C S-matrix in general Chern-Simons bosonic vector theory } & 21\end{array}$

\section{Introduction}

Three dimensional vector model gauged by Chern-Simons interaction (Chern-Simons vector model) has turned out to be an intersection of various subjects in theoretical physics. An initiative study was done in [1,2], where it was conjectured that Chern-Simons vector model inherits large $N$ solubility of vector model in the 't Hooft large $N$ limit and becomes a conformal field theory (CFT) dual to parity-violated higher-spin gravity theory in $\mathrm{AdS}_{4}[3]$ by extending the conjecture of vector model/higher-spin theory [4]. (See [5] for its supersymmetric extension.)

Large $N$ exact computations of three point correlation functions in Chern-Simons vector models were done $[6,7]$ in accordance with general study of correlators including parity non-invariant contact terms of the class of three dimensional CFT [8-10]. (See also $[11,12]$.) Analysis of correlation functions indicated that there exists a bosonization duality between a pair of a bosonic Chern-Simons vector model and a fermionic one as a generalization of level-rank duality known in pure Chern-Simons theory by including vector matter fields. This novel duality was confirmed from the large $N$ thermal free energy of Chern-Simons vector models [13-17]. (See also [1, 18-21].) Evidence for this duality to hold in the renormalization group (RG) flow was provided in [15, 17, 22], which indicated that this bosonization duality in three dimensions is obtained by continuous deformation from supersymmetric duality known as the Seiberg-like duality [23, 24]. (See also [25-29] for studies of other aspects.) 
Three dimensional bosonization duality was uncovered from large $N$ exact results of scattering amplitudes at the level of elementary particles [30, 31]. (See also [32].) It turned out that the scattering amplitude in the S-channel computed from Euclidean theory via Wick rotation becomes non-unitary [30], which was also observed in the original AharonovBohm scattering. The non-unitarity problem in the non-relativistic case was resolved by carefully taking into account the singular contribution in forward scattering [33]. The resolution of non-unitarity puzzle in the relativistic case was similarly developed by taking care of the forward scattering so as to restore unitarity [30]. Then it was shown that the improved S-matrix exhibits bosonization duality, which just exchanges bosonic particles in one theory with fermionic ones in the dual one. By combining this with the fact that a bosonic or fermionic particle interacting with a Chern-Simons gauge field acquires anyon statistics $[34,35]$, the bosonization in three dimensions can be naturally understood as a duality between two theories of anyons.

Along these lines this paper aims at performing the exact large $N$ calculation of scattering amplitudes and confirming the duality in a general Chern-Simons vector model, which includes the double and triple trace terms in the bosonic Chern-Simons vector model or quadratic and cubic ones of the auxiliary field in the fermionic one. The bosonization duality is expected to hold for general Chern-Simons vector models since these models connect the regular Chern-Simons vector models and the critical ones by the RG flow. Indeed the duality in the general case was confirmed in an exact large $N$ calculation of the thermal free energy [17]. The calculation of the S-matrix on the bosonic side is straightforward by adding the contribution of the triple trace coupling into that in [30], while the calculation in the fermionic side is rather non-trivial since the interaction of the auxiliary field generates ultra-violet (UV) divergent integrals as fermion loops in the Gross-Neveu model [36, 37], which are non-renormalizable in the sense of a weak coupling expansion and have to be regularized suitably in the $1 / N$ expansion.

The rest of this paper is organized as follows. In section 2 we set up the system and perform some preliminary large $N$ analysis. In section 3 we compute the four point function of the fermionic field exactly in the light-cone gauge with a specific momentum frame. By applying the LSZ formula to the four point function we determine the S-matrix in the T-channel in section 4.1, and that in S-channels with taking account of non-analytic contribution in the forward scattering in section 4 . Section 5 is devoted to conclusion and discussion. In appendix we provide details about the regularization A, construct asymptotic states of scattering process $\mathrm{B}$, and give a brief derivation of scattering amplitudes in general Chern-Simons bosonic vector models $\mathrm{C}$.

\section{General Chern-Simons fermion vector model}

In this section we perform preliminary analysis on general $\mathrm{U}(N)_{k_{F}}$ Chern-Simons fermion vector model in the 't Hooft large $N$ limit, $N, k_{F} \rightarrow \infty$ with $\lambda:=N / k_{F}$ fixed. ${ }^{1}$ Lagrangian

\footnotetext{
${ }^{1}$ We basically use the same convention adopted in [17]. Under the convention the 't Hooft coupling constant is bounded so that $|\lambda| \leq 1$. Especially upon encountering divergent integrals we regularize them by dimensional regularization in the way described in [18].
} 
Figure 1. Feynman rule involving the auxiliary field $\sigma_{F}$ is depicted. The solid and dotted lines represent the fermion and the auxiliary field, respectively. From the left, the diagrams describe tadpole, tree-level propagator, Yukawa interaction with fermion, and cubic self-interaction.

of general $\mathrm{U}(N)_{k_{F}}$ Chern-Simons fermion vector model is defined by using an auxiliary field $\sigma_{F}$ as

$$
\begin{aligned}
\mathcal{L}_{F}= & i \varepsilon^{\mu \nu \rho} \frac{k_{F}}{4 \pi} \operatorname{Tr}\left(A_{\mu} \partial_{\nu} A_{\rho}-\frac{2 i}{3} A_{\mu} A_{\nu} A_{\rho}\right)+\bar{\psi} \gamma_{\mu} D^{\mu} \psi \\
& +\sigma_{F}\left(\bar{\psi} \psi-\frac{k_{F}}{4 \pi} y_{2}^{2}\right)-\frac{k_{F}}{4 \pi} y_{4} \sigma_{F}^{2}+\frac{k_{F}}{4 \pi} y_{6} \sigma_{F}^{3}
\end{aligned}
$$

where

$$
D_{\mu} \psi=\left(\partial_{\mu}-i A_{\mu}\right) \psi, \quad D_{\mu} \bar{\psi}=\left(\partial_{\mu} \bar{\psi}+i \bar{\psi} A_{\mu}\right) .
$$

Here we have dropped the gauge, spinor and space indices. For convenience we depict Feynman rules involving the auxiliary field in figure 1. This theory is not renormalizable in the weak coupling expansion whereas it is in the $1 / N$ expansion. In particular, when the cubic self-interaction of the auxiliary field vanishes this system is the same as the three dimensional Gross-Neveu model [36] gauged with Chern-Simons interactions. In contrast to the fact that the two dimensional Gross-Neveu model is asymptotically free, the three dimensional one has an ultra-violet fixed point [37]. In the current setup the critical theory is obtained by a limit such that $y_{2}^{2}, y_{4}, y_{6} \rightarrow 0$. Note that the auxiliary field has odd parity since the Gross-Neveu model is classically parity invariant.

The exact propagator of the fermionic field in the large $N$ limit was already computed in [17]. The result reads

$$
\left\langle\psi^{m}(p) \bar{\psi}_{n}\left(-p^{\prime}\right)\right\rangle=\delta_{n}^{m}(2 \pi)^{3} \delta^{3}\left(p-p^{\prime}\right) \alpha_{F}(p), \quad \alpha_{F}(p)=\frac{1}{i \gamma^{\mu} p_{\mu}+\Sigma_{F}(p)} .
$$

Here $-\Sigma_{F}(p)$ is the fermion 1PI self energy given by

$$
\begin{aligned}
& \Sigma_{F}(p)=\Sigma_{I}(p)+\Sigma_{+}(p) \gamma^{+}, \\
& \Sigma_{I}(p)=\lambda \sqrt{p_{s}^{2}+c_{F}^{2}}+(\operatorname{sgn}(\lambda)-\lambda) c_{F}, \\
& \Sigma_{+}(p)=i p_{+} \frac{c_{F}^{2}-\Sigma_{I}(p)^{2}}{p_{s}^{2}},
\end{aligned}
$$

where $p_{ \pm}:=\frac{p_{1} \pm i p_{2}}{\sqrt{2}}, p_{s}:=2 p_{+} p_{-}$and $c_{F}$ is the physical fermion mass determined by the gap equation

$$
\left(1-3 y_{6}\right)(\operatorname{sgn}(\lambda)-\lambda)^{2} c_{F}^{2}+2 y_{4}(\operatorname{sgn}(\lambda)-\lambda) c_{F}+y_{2}^{2}-c_{F}^{2}=0 .
$$

Note that we consider the cases where $c_{F}>0$. 


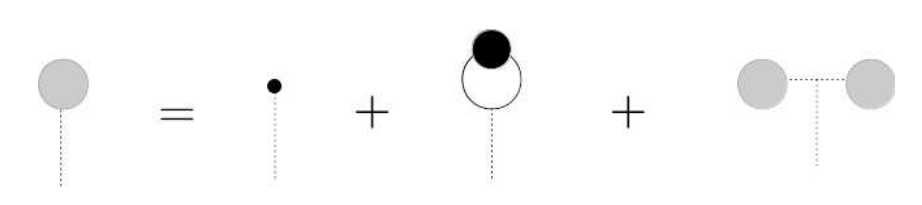

Figure 2. The bootstrap diagram for the exact tadpole is drawn. Here the black node represents the exact fermion propagator and the gray node describes the exact tadpole diagram.

For later convenience we determine the exact tadpole diagram contribution. The tadpole diagram satisfies the bootstrap diagram depicted in figure 2 , which reads

$$
T=\frac{k_{F}}{4 \pi} y_{2}^{2}-N \int \frac{d^{3} p}{(2 \pi)^{3}}\left(-\operatorname{tr} \frac{1}{i \gamma^{\mu} p_{\mu}+\Sigma_{F}(p)}\right)-3 \frac{k_{F}}{4 \pi} y_{6} \times\left(\frac{1}{-2 \frac{k_{F}}{4 \pi} y_{4}} T\right)^{2},
$$

where $T$ is the contribution of the tadpole diagram. The equation (2.6) is quadratic so there are in general two solutions. We restrict our interest to the region of coupling constants where

$$
\operatorname{sgn}\left(y_{4}\right)=\operatorname{sgn}\left(y_{4}+3 c_{F} y_{6}(\lambda-\operatorname{sgn}(\lambda))\right)
$$

whose physical implication will be discussed in the next section. We choose a solution which has the correct perturbative continuation so that $T \rightarrow \frac{k_{F}}{4 \pi} y_{2}^{2}$ with $y_{6}, \lambda \rightarrow 0$ :

$$
T=\frac{-k_{F} y_{4}^{2}}{6 \pi y_{6}}\left(1-\sqrt{1+\frac{3 y_{6}}{y_{4}^{2}}\left(c_{F}^{2}(|\lambda|-2)|\lambda|+y_{2}^{2}\right)}\right)=\frac{k_{F}}{2 \pi} c_{F} y_{4}(\lambda-\operatorname{sgn}(\lambda)),
$$

where in the second equality we used the gap equation (2.5) to remove $y_{2}^{2}$.

\section{Four point function of fermions}

In this section we compute the fermion four point function in the large $N$ limit. Since the amputated connected four point function is equivalent to the four point vertex, we compute the latter. In particular we compute the four point vertex of the large $N$ form such that

$$
-\frac{1}{2} \int \frac{d^{3} p}{(2 \pi)^{3}} \frac{d^{3} k}{(2 \pi)^{3}} \frac{d^{3} q}{(2 \pi)^{3}} \bar{\psi}_{\alpha^{\prime}, m}(-p-q) \psi^{\beta^{\prime}, m}(p) F_{\beta^{\prime}}^{\alpha^{\prime}}(p, k ; q) \bar{\psi}_{\beta, n}(-k) \psi^{\alpha, n}(k+q),
$$

where spinor indices, which are denoted by Greek letters, are explicitly restored. In the large $N$ limit the bootstrap diagram of the four point vertex is simplified to be selfconsistent without any other higher point vertices and given by the ladder diagram in figure 3. From this figure the Schwinger-Dyson equation for the four point vertex reads

$$
\begin{aligned}
& F^{\alpha^{\prime}{ }^{\beta}{ }_{\alpha}(p, k ; q)=F^{0 \alpha^{\prime}}{ }_{\beta^{\prime}}{ }_{\alpha}(p, k ; q)}
\end{aligned}
$$

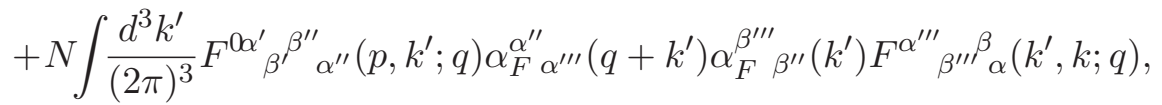

where $F^{0}$ describes the contribution of the ladder piece given by

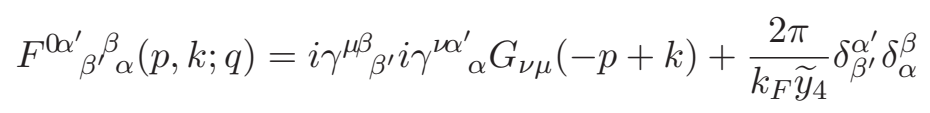




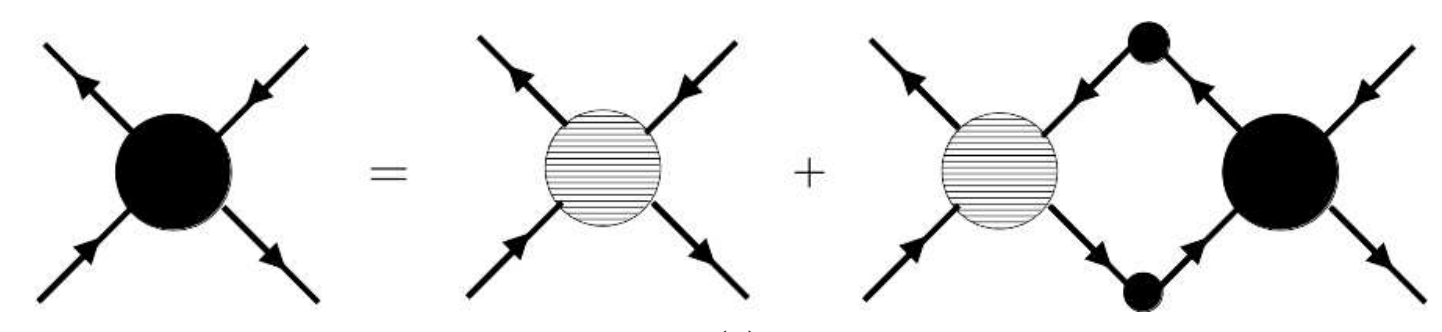

(a)
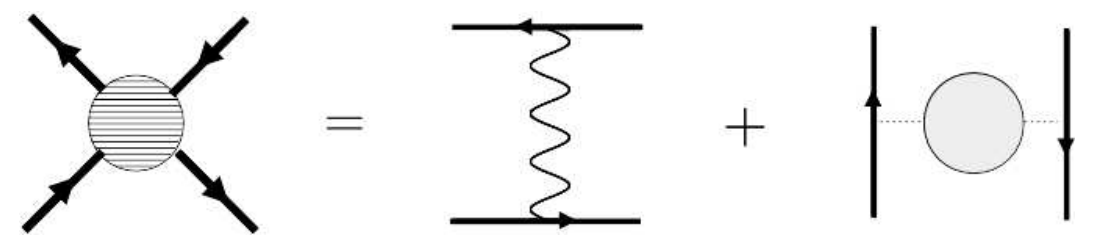

(b)

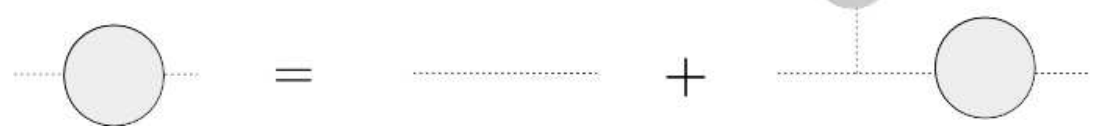

(c)

Figure 3. (a) shows the exact bootstrap diagram for the fermion four point function in the large $N$ limit. Here the black bubble with two or four lines represents the exact two or four point function, respectively, and the striped bubble represents the ladder bar of this ladder diagram. (b) shows the definition of the ladder bar, in which the striped bubble is given by summation of one gluon exchange diagram and that with fermionic lines connected by the auxiliary fields with a gray bubble, which is defined as the two point function whose 1PI diagram is given only by the tadpole as depicted in (c).

with

$$
\begin{aligned}
G_{\mu \nu}(p) & =\frac{2 \pi}{i k_{F} p_{-}}\left(\delta_{\mu,+} \delta_{\nu, 3}-\delta_{\mu, 3} \delta_{\nu,+}\right), \\
\widetilde{y}_{4} & =y_{4}+3 c_{F} y_{6}(\lambda-\operatorname{sgn}(\lambda)) .
\end{aligned}
$$

We give some comments. The first term in (3.3) is the contribution of one gluon exchange diagram depicted in figure 3(b). $G_{\mu \nu}(p)$ is the Chern-Simons gauge propagator, which is given as (3.4) in the light-cone gauge, where the matter loop correction is subleading in the $1 / N$ expansion. The second term in (3.3) describes the contribution from the auxiliary field. It is given by the exact two point correlator of the auxiliary field excluding fermion loops, which are already taken into account in the main bootstrap equation (3.2) as described in figure $3(\mathrm{a})$. Denoting the contribution by $\frac{-2 \pi}{k_{F} \widetilde{y}_{4}}$, we can determine it by the bootstrap diagram given by figure $3(\mathrm{c})$, which reads

$$
\frac{-2 \pi}{k_{F} \widetilde{y}_{4}}=\frac{-2 \pi}{k_{F} y_{4}}+\left(-3 ! \frac{k_{F}}{4 \pi} y_{6}\right) \times\left(\frac{-2 \pi}{k_{F} y_{4}}\right) \times T \times\left(\frac{-2 \pi}{k_{F} y_{4}}\right) \times\left(\frac{-2 \pi}{k_{F} \widetilde{y}_{4}}\right)
$$

where $T$ is the contribution of the exact tadpole diagram given by (2.8). Solving (3.6) gives (3.5). Therefore, in the coupling region specified by (2.7) the quantum two point 
correlator of the scalar field (excluding fermion loops) has the same sign as the one at tree level, which may suggest that the current system is reflection positive or stable in the quantum mechanical sense under the coupling region (2.7).

To solve the bootstrap equation (3.2) it is convenient to transform the spinor indices into the vector ones by using the gamma matrices so that

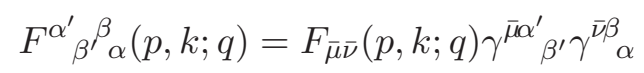

where $\bar{\mu}=1,2,3, I$ and $\gamma^{I}=I$. Plugging this into the equation above and performing the straight-forward gamma gymnastics we find that the above equation reduces to two sets of consistent integral equations. One set is on $F_{I I}$ and $F_{+I}$, which is given by

$$
\begin{aligned}
F_{I I}(p, k)= & \frac{2 \pi}{k_{F} \widetilde{y}_{4}}-2 \pi i \lambda \int \frac{d^{3} k^{\prime}}{(2 \pi)^{3}} \frac{2\left(2 F_{+I}\left(k^{\prime}, k\right) k_{-}^{2}+F_{I I}\left(k^{\prime}, k\right) k_{-}^{\prime}\left(2 i \Sigma_{I}\left(k^{\prime}\right)-q_{3}\right)\right)}{\left(-p+k^{\prime}\right)_{-}\left(k^{\prime 2}+c_{F}^{2}\right)\left(\left(k^{\prime}+q\right)^{2}+c_{F}^{2}\right)} \\
& +\frac{2 \pi \lambda}{\widetilde{y}_{4}} \int \frac{d^{3} k^{\prime}}{(2 \pi)^{3}} \frac{-2\left(F_{+I}\left(k^{\prime}, k\right) k_{-}^{\prime}\left(2 i \Sigma_{I}\left(k^{\prime}\right)+q_{3}\right)+F_{I I}\left(k^{\prime}, k\right)\left(k_{s}^{\prime 2}+c_{F}^{2}-2 \Sigma_{I}^{2}\left(k^{\prime}\right)+k_{3}^{\prime 2}+q_{3} k_{3}^{\prime}\right)\right.}{\left(k^{\prime 2}+c_{F}^{2}\right)\left(\left(k^{\prime}+q\right)^{2}+c_{F}^{2}\right)}, \\
F_{+I}(p, k)= & \frac{-\pi i}{2 k_{F}(-p+k)_{-}}-2 \pi i \lambda \times \\
& \times \int \frac{d^{3} k^{\prime}}{(2 \pi)^{3}} \frac{-2\left(F_{+I}\left(k^{\prime}, k\right) k_{-}^{\prime}\left(2 i \Sigma_{I}\left(k^{\prime}\right)+q_{3}\right)+F_{I I}\left(k^{\prime}, k\right)\left(k_{s}^{\prime 2}+c_{F}^{2}-2 \Sigma_{I}^{2}\left(k^{\prime}\right)+k_{3}^{\prime 2}+q_{3} k_{3}^{\prime}\right)\right.}{\left(-p+k^{\prime}\right)-\left(k^{\prime 2}+c_{F}^{2}\right)\left(\left(k^{\prime}+q\right)^{2}+c_{F}^{2}\right)}
\end{aligned}
$$

where we omit the third variable $q$ in the scattering function. The other set is on $F_{I+}$ and $F_{++}$, which is given by

$$
\begin{aligned}
F_{I+}(p, k)= & \frac{2 \pi i}{k_{F}(-p+k)_{-}}-2 \pi i \lambda \int \frac{d^{3} k^{\prime}}{(2 \pi)^{3}} \frac{2\left(2 F_{++}\left(k^{\prime}, k\right) k_{-}^{2}+F_{I+}\left(k^{\prime}, k\right) k_{-}^{\prime}\left(2 i \Sigma_{I}\left(k^{\prime}\right)-q_{3}\right)\right)}{\left(-p+k^{\prime}\right)-\left(k^{\prime 2}+c_{F}^{2}\right)\left(\left(k^{\prime}+q\right)^{2}+c_{F}^{2}\right)} \\
& +\frac{2 \pi \lambda}{\widetilde{y}_{4}} \int \frac{d^{3} k^{\prime}}{(2 \pi)^{3}} \frac{-2\left(F_{++}\left(k^{\prime}, k\right) k_{-}^{\prime}\left(2 i \Sigma_{I}\left(k^{\prime}\right)+q_{3}\right)+F_{I+}\left(k^{\prime}, k\right)\left(k_{s}^{\prime 2}+c_{F}^{2}-2 \Sigma_{I}^{2}\left(k^{\prime}\right)+k_{3}^{\prime 2}+q_{3} k_{3}^{\prime}\right)\right.}{\left(k^{\prime 2}+c_{F}^{2}\right)\left(\left(k^{\prime}+q\right)^{2}+c_{F}^{2}\right)}, \\
F_{++}(p, k)= & 4 \pi i \lambda \int \frac{d^{3} k^{\prime}}{(2 \pi)^{3}} \frac{F_{++}\left(k^{\prime}, k\right) k_{-}^{\prime}\left(2 i \Sigma_{I}\left(k^{\prime}\right)+q_{3}\right)+F_{I I}\left(k^{\prime}, k\right)\left(k_{s}^{\prime 2}+c_{F}^{2}-2 \Sigma_{I}^{2}\left(k^{\prime}\right)+k_{3}^{\prime 2}+q_{3} k_{3}^{\prime}\right)}{\left(-p+k^{\prime}\right)-\left(k^{\prime 2}+c_{F}^{2}\right)\left(\left(k^{\prime}+q\right)^{2}+c_{F}^{2}\right)} .
\end{aligned}
$$

These integral equations can be solved exactly in a specific momentum frame such that

$$
q_{ \pm}=\frac{q_{1} \pm i q_{2}}{\sqrt{2}}=0
$$

This frame matches the center of mass frame in the S-channel scattering, which is investigated in the next section. Then let us make the following ansatz:

$$
\begin{gathered}
F_{I I}(p, k)=\frac{p_{-}}{(-p+k)_{-}} B_{1}\left(p_{s}, k_{s}\right)+B_{2}\left(p_{s}, k_{s}\right), \\
F_{+I}(p, k)=\frac{1}{(-p+k)_{-}} A_{1}\left(p_{s}, k_{s}\right)+\frac{1}{p_{-}} A_{2}\left(p_{s}, k_{s}\right),
\end{gathered}
$$

and

$$
\begin{aligned}
F_{I+}(p, k) & =\frac{1}{(-p+k)_{-}} B_{3}\left(p_{s}, k_{s}\right)+\frac{1}{k_{-}} B_{4}\left(p_{s}, k_{s}\right), \\
F_{++}(p, k) & =\frac{1}{p_{-}(-p+k)_{-}} A_{3}\left(p_{s}, k_{s}\right)+\frac{1}{p_{-} k_{-}} A_{4}\left(p_{s}, k_{s}\right) .
\end{aligned}
$$

Plugging this ansatz into the equation bove and performing the integral for $k_{3}^{\prime}$ and the angular part of $k_{-}^{\prime}$ we obtain 


$$
\begin{aligned}
B_{1}\left(p_{s}, k_{s}\right)= & -4 \pi i \lambda \int_{h_{p}}^{h_{k}} \frac{d h}{(2 \pi)} \frac{2 A_{1}+B_{1}\left(2 i \Sigma_{I}-q_{3}\right)}{4 h^{2}+q_{3}^{2}} \\
A_{1}\left(p_{s}, k_{s}\right)= & \frac{-2 \pi i}{k_{F}}+4 \pi i \lambda \int_{h_{p}}^{h_{k}} \frac{d h}{(2 \pi)} \frac{A_{1}\left(2 i \Sigma_{I}+q_{3}\right)+2 B_{1}\left(h^{2}-\Sigma_{I}^{2}\right)}{4 h^{2}+q_{3}^{2}} \\
B_{2}\left(p_{s}, k_{s}\right)= & \frac{2 \pi}{k_{F} \widetilde{y}_{4}}-4 \pi i \lambda\left(-\int_{h_{k}}^{\infty} \frac{d h}{(2 \pi)} \frac{2 A_{1}+B_{1}\left(2 i \Sigma_{I}-q_{3}\right)}{4 h^{2}+q_{3}^{2}}+\int_{h_{p}}^{\infty} \frac{d h}{(2 \pi)} \frac{2 A_{2}+B_{2}\left(2 i \Sigma_{I}-q_{3}\right)}{4 h^{2}+q_{3}^{2}}\right) \\
& -\frac{4 \pi \lambda}{\widetilde{y}_{4}}\left(-\int_{h_{k}}^{\infty} \frac{d h}{(2 \pi)} \frac{A_{1}\left(2 i \Sigma_{I}+q_{3}\right)+2 B_{1}\left(h^{2}-\Sigma_{I}^{2}\right)}{4 h^{2}+q_{3}^{2}}\right. \\
& \left.+\int_{h_{0}}^{\infty} \frac{d h}{(2 \pi)} \frac{A_{2}\left(2 i \Sigma_{I}+q_{3}\right)+2 B_{2}\left(h^{2}-\Sigma_{I}^{2}\right)}{4 h^{2}+q_{3}^{2}}\right) \\
A_{2}\left(p_{s}, k_{s}\right)= & -4 \pi i \lambda \int_{h_{0}}^{h_{p}} \frac{d h}{(2 \pi)} \frac{A_{2}\left(2 i \Sigma_{I}+q_{3}\right)+2 B_{2}\left(h^{2}-\Sigma_{I}^{2}\right)}{4 h^{2}+q_{3}^{2}}
\end{aligned}
$$

and

$$
\begin{aligned}
B_{3}\left(p_{s}, k_{s}\right)= & \frac{2 \pi i}{k_{F}}-4 \pi i \lambda \int_{h_{p}}^{h_{k}} \frac{d h}{(2 \pi)} \frac{2 A_{3}+B_{3}\left(2 i \Sigma_{I}-q_{3}\right)}{4 h^{2}+q_{3}^{2}} \\
A_{3}\left(p_{s}, k_{s}\right)= & 4 \pi i \lambda \int_{h_{p}}^{h_{k}} \frac{d h}{(2 \pi)} \frac{A_{3}\left(2 i \Sigma_{I}+q_{3}\right)+2 B_{3}\left(h^{2}-\Sigma_{I}^{2}\right)}{4 h^{2}+q_{3}^{2}} \\
B_{4}\left(p_{s}, k_{s}\right)= & -4 \pi i \lambda \int_{h_{p}}^{\infty} \frac{d h}{(2 \pi)} \frac{2 A_{4}+B_{4}\left(2 i \Sigma_{I}-q_{3}\right)}{4 h^{2}+q_{3}^{2}}-\frac{4 \pi \lambda}{\widetilde{y}_{4}}\left(\int_{h_{0}}^{h_{k}} \frac{d h}{(2 \pi)} \frac{A_{3}\left(2 i \Sigma_{I}+q_{3}\right)+2 B_{3}\left(h^{2}-\Sigma_{I}^{2}\right)}{4 h^{2}+q_{3}^{2}}\right. \\
& \left.+\int_{h_{0}}^{\infty} \frac{d h}{(2 \pi)} \frac{A_{4}\left(2 i \Sigma_{I}+q_{3}\right)+2 B_{4}\left(h^{2}-\Sigma_{I}^{2}\right)}{4 h^{2}+q_{3}^{2}}\right) \\
A_{4}\left(p_{s}, k_{s}\right)=-4 \pi i \lambda & \left(\int_{h_{0}}^{h_{k}} \frac{d h}{(2 \pi)} \frac{A_{3}\left(2 i \Sigma_{I}+q_{3}\right)+2 B_{3}\left(h^{2}-\Sigma_{I}^{2}\right)}{4 h^{2}+q_{3}^{2}}\right. \\
& \left.+\int_{h_{0}}^{h_{p}} \frac{d h}{(2 \pi)} \frac{A_{4}\left(2 i \Sigma_{I}+q_{3}\right)+2 B_{4}\left(h^{2}-\Sigma_{I}^{2}\right)}{4 h^{2}+q_{3}^{2}}\right)
\end{aligned}
$$

where we set $h_{p}:=\sqrt{p_{s}^{2}+c_{F}^{2}}$, and in the right-hand side $\Sigma_{I}=\lambda h+(\operatorname{sgn}(\lambda)-\lambda) c_{F}$ and $A_{i}=A_{i}\left(k_{s}^{\prime}, k_{s}\right), B_{i}=B_{i}\left(k_{s}^{\prime}, k_{s}\right)$ with $h=h_{k^{\prime}}, i=1,2,3,4$.

In order to solve this let us take the derivative with respect to $h_{p}$ on both sides. Then we obtain

$$
\begin{aligned}
& \frac{\partial B_{i}}{\partial h_{p}}=2 i \lambda \frac{2 A_{i}+B_{i}\left(2 i \Sigma_{I}(p)-q_{3}\right)}{4 h_{p}^{2}+q_{3}^{2}} \\
& \frac{\partial A_{i}}{\partial h_{p}}=-2 i \lambda \frac{A_{i}\left(2 i \Sigma_{I}(p)+q_{3}\right)+2 B_{i}\left(h_{p}^{2}-\Sigma_{I}(p)^{2}\right)}{4 h_{p}^{2}+q_{3}^{2}}
\end{aligned}
$$

for all $i=1,2,3,4$. From these two equations one can derive

$$
\frac{\partial}{\partial h_{p}}\left(B_{i}\left(2 i \Sigma+q_{3}\right)+2 A_{i}\right)=0 .
$$

Thus $A_{i}$ can be related to $B_{i}$ by

$$
A_{i}\left(p_{s}, k_{s}\right)=-B_{i}\left(p_{s}, k_{s}\right)\left(i \Sigma(p)+\frac{1}{2} q_{3}\right)+\alpha_{i}
$$


where $\alpha_{i}$ is an unknown function independent of $p_{s}$. Plugging this back into (3.25) we find

$$
\frac{\partial B_{i}}{\partial h_{p}}=2 i \lambda \frac{-2 q_{3} B_{i}+2 \alpha_{i}}{4 h_{p}^{2}+q_{3}^{2}} .
$$

This can be solved as

$$
B_{i}\left(p_{s}, k_{s}\right)=\frac{\alpha_{i}}{q_{3}}+\beta_{i} f\left(p_{s}\right)
$$

where $\beta_{i}$ is another integration constant independent of $p_{s}$ and $f\left(p_{s}\right)$ is defined by

$$
f\left(p_{s}\right):=\exp \left[-2 i \lambda \arctan \frac{2 \sqrt{p_{s}^{2}+c_{F}^{2}}}{q_{3}}\right] .
$$

The integration constants $\alpha_{i}, \beta_{i}$ can be determined so as to satisfy the following conditions derived from the integral equations.

$$
\begin{aligned}
\lim _{p_{s} \rightarrow k_{s}} B_{1}\left(p_{s}, k_{s}\right)= & 0, \\
\lim _{p_{s} \rightarrow k_{s}} A_{1}\left(p_{s}, k_{s}\right)= & \frac{-2 \pi i}{k_{F}}, \\
\lim _{p_{s} \rightarrow \infty} B_{2}\left(p_{s}, k_{s}\right)= & \frac{2 \pi}{k_{F} \widetilde{y}_{4}}+4 \pi i \lambda \int_{h_{k}}^{\infty} \frac{d h}{(2 \pi)} \frac{2 A_{1}+B_{1}\left(2 i \Sigma_{I}-q_{3}\right)}{4 h^{2}+q_{3}^{2}} \\
& -\frac{4 \pi \lambda}{\widetilde{y}_{4}}\left(-\int_{h_{k}}^{\infty} \frac{d h}{(2 \pi)} \frac{A_{1}\left(2 i \Sigma_{I}+q_{3}\right)+2 B_{1}\left(h^{2}-\Sigma_{I}^{2}\right)}{4 h^{2}+q_{3}^{2}}\right. \\
& \left.+\int_{h_{0}}^{\infty} \frac{d h}{(2 \pi)} \frac{A_{2}\left(2 i \Sigma_{I}+q_{3}\right)+2 B_{2}\left(h^{2}-\Sigma_{I}^{2}\right)}{4 h^{2}+q_{3}^{2}}\right),
\end{aligned}
$$

$\lim _{p_{s} \rightarrow 0} A_{2}\left(p_{s}, k_{s}\right)=0$,

and

$$
\begin{aligned}
\lim _{p_{s} \rightarrow k_{s}} B_{3}\left(p_{s}, k_{s}\right) & =\frac{2 \pi i}{k_{F}}, \\
\lim _{p_{s} \rightarrow k_{s}} A_{3}\left(p_{s}, k_{s}\right) & =0, \\
\lim _{p_{s} \rightarrow \infty} B_{4}\left(p_{s}, k_{s}\right)=-\frac{4 \pi \lambda}{\widetilde{y}_{4}}\left(\int_{h_{0}}^{h_{k}} \frac{d h}{(2 \pi)} \frac{A_{3}\left(2 i \Sigma_{I}+q_{3}\right)+2 B_{3}\left(h^{2}-\Sigma_{I}^{2}\right)}{4 h^{2}+q_{3}^{2}}\right. & \\
& \left.+\int_{h_{0}}^{\infty} \frac{d h}{(2 \pi)} \frac{A_{4}\left(2 i \Sigma_{I}+q_{3}\right)+2 B_{4}\left(h^{2}-\Sigma_{I}^{2}\right)}{4 h^{2}+q_{3}^{2}}\right), \\
\lim _{p_{s} \rightarrow 0} A_{4}\left(p_{s}, k_{s}\right) & =-4 \pi i \lambda \int_{h_{0}}^{h_{k}} \frac{d h}{(2 \pi)} \frac{A_{3}\left(2 i \Sigma_{I}+q_{3}\right)+2 B_{3}\left(h^{2}-\Sigma_{I}^{2}\right)}{4 h^{2}+q_{3}^{2}} .
\end{aligned}
$$

By using (3.33), (3.32), (3.37), (3.36), $\alpha_{1}, \beta_{1}, \alpha_{3}, \beta_{3}$ can be easily determined as

$$
\begin{aligned}
\alpha_{1} & =\frac{-2 \pi i}{k_{F}}, & \beta_{1} & =\frac{2 \pi i}{k_{F} q_{3} f\left(k_{s}\right)}, \\
\alpha_{3} & =\left(2 i \Sigma_{I}\left(k_{s}\right)+q_{3}\right) \frac{\pi i}{k_{F}}, & \beta_{3} & =\frac{\pi i\left(-2 i \Sigma_{I}\left(k_{s}\right)+q_{3}\right)}{k_{F} q_{3} f\left(k_{s}\right)} .
\end{aligned}
$$


On the other hand, the right-hand sides in (3.34), (3.38) contain divergent integrals, which need regularization. This divergence originates in the non-renormalizable interaction in the sense of power counting in (2.1). We regularize the divergence by dimensional regularization demonstrated in detail in [18], which is skipped here and move it to appendix A. After the regularization we can determine $\alpha_{2}, \beta_{2}, \alpha_{4}, \beta_{4}$ by using (3.35), (3.34), (3.39), (3.38) as

$$
\alpha_{i}=\frac{(\text { num })_{\alpha_{i}}}{(\text { den })_{\alpha_{i}}}, \quad \beta_{i}=\frac{(\text { num })_{\beta_{i}}}{(\text { den })_{\beta_{i}}}
$$

where

$$
\begin{aligned}
(\text { num })_{\alpha_{2}}= & 2 \pi f(0)\left(q_{3}+2 i \Sigma_{I}(0)\right)\left(f\left(k_{s}\right)\left(2 c_{F}(\lambda-\operatorname{sgn}(\lambda))-i q_{3}-2 \widetilde{y}_{4}\right)\right. \\
& \left.+f(\infty)\left(-2 c_{F}(\lambda-\operatorname{sgn}(\lambda))-i q_{3}+2 \widetilde{y}_{4}\right)\right) \\
(\text { den })_{\alpha_{2}}= & k_{F} f\left(k_{s}\right)\left\{f(\infty)\left(2 \Sigma_{I}(0)+i q_{3}\right)\left(2 c_{F}(\lambda-\operatorname{sgn}(\lambda))+i q_{3}-2 \widetilde{y}_{4}\right)\right. \\
& \left.+f(0)\left(c_{F}\left(-4(\lambda-\operatorname{sgn}(\lambda)) \Sigma_{I}(0)+2 i(\lambda+\operatorname{sgn}(\lambda)) q_{3}\right)+q_{3}^{2}-2 i q_{3}\left(\Sigma_{I}(0)+\widetilde{y}_{4}\right)+4 \Sigma_{I}(0) \widetilde{y}_{4}\right)\right\} \\
(\text { num })_{\beta_{2}}= & -2 \pi\left(q_{3}-2 i \Sigma_{I}(0)\right)\left(f\left(k_{s}\right)\left(-2 c_{F}(\lambda-\operatorname{sgn}(\lambda))+i q_{3}+2 \widetilde{y}_{4}\right)\right. \\
& \left.+f(\infty)\left(2 c_{F}(\lambda-\operatorname{sgn}(\lambda))+i q_{3}-2 \widetilde{y}_{4}\right)\right) \\
(\text { den })_{\beta_{2}}= & k_{F} q_{3} f\left(k_{s}\right)\left\{f(\infty)\left(2 \Sigma_{I}(0)+i q_{3}\right)\left(2 c_{F}(\lambda-\operatorname{sgn}(\lambda))+i q_{3}-2 \widetilde{y}_{4}\right)\right. \\
& \left.+f(0)\left(c_{F}\left(-4(\lambda-\operatorname{sgn}(\lambda)) \Sigma_{I}(0)+2 i(\lambda+\operatorname{sgn}(\lambda)) q_{3}\right)+q_{3}^{2}-2 i q_{3}\left(\Sigma_{I}(0)+\widetilde{y}_{4}\right)+4 \Sigma_{I}(0) \widetilde{y}_{4}\right)\right\} \\
(\text { num })_{\alpha_{4}}= & \pi\left(f\left(k_{s}\right)\left(q_{3}-2 i \Sigma_{I}(0)\right)\left(q_{3}+2 i \Sigma_{I}\left(k_{s}\right)\right)-f(0)\left(q_{3}+2 i \Sigma_{I}(0)\right)\left(q_{3}-2 i \Sigma_{I}\left(k_{s}\right)\right)\right) \times \\
& \times\left(2\left(c_{F}-\Sigma_{I}(0)\right) f(0)+f(\infty)\left(2 c_{F}(\lambda-\operatorname{sgn}(\lambda))+i q_{3}-2 \widetilde{y}_{4}\right)\right), \\
(\text { den })_{\alpha_{4}}= & k_{F} f\left(k_{s}\right)\left\{f(\infty)\left(2 \Sigma_{I}(0)+i q_{3}\right)\left(2 c_{F}(\lambda-\operatorname{sgn}(\lambda))+i q_{3}-2 \widetilde{y}_{4}\right)\right. \\
& \left.+f(0)\left(c_{F}\left(-4(\lambda-\operatorname{sgn}(\lambda)) \Sigma_{I}(0)+2 i(\lambda+\operatorname{sgn}(\lambda)) q_{3}\right)+q_{3}^{2}-2 i q_{3}\left(\Sigma_{I}(0)+\widetilde{y}_{4}\right)+4 \Sigma_{I}(0) \widetilde{y}_{4}\right)\right\} \\
(\text { num })_{\beta_{4}}= & \pi\left(-2\left(-c_{F} \lambda+\Sigma_{I}(0)+\widetilde{y}_{4}\right)-i q_{3}\right)\left(f(0)\left(q_{3}+2 i \Sigma_{I}(0)\right)\left(q_{3}-2 i \Sigma_{I}\left(k_{s}\right)\right)\right. \\
& \left.-f\left(k_{s}\right)\left(q_{3}-2 i \Sigma_{I}(0)\right)\left(q_{3}+2 i \Sigma_{I}\left(k_{s}\right)\right)\right), \\
(\text { den })_{\beta_{4}}= & k_{F} q_{3} f\left(k_{s}\right)\left\{f(\infty)\left(2 \Sigma_{I}(0)+i q_{3}\right)\left(2 c_{F}(\lambda-\operatorname{sgn}(\lambda))+i q_{3}-2 \widetilde{y}_{4}\right)\right. \\
& \left.+f(0)\left(c_{F}\left(-4(\lambda-\operatorname{sgn}(\lambda)) \Sigma_{I}(0)+2 i(\lambda+\operatorname{sgn}(\lambda)) q_{3}\right)+q_{3}^{2}-2 i q_{3}\left(\Sigma_{I}(0)+\widetilde{y}_{4}\right)+4 \Sigma_{I}(0) \widetilde{y}_{4}\right)\right\} .
\end{aligned}
$$

A few comments are in order. Firstly, by taking the limit $\widetilde{y}_{4} \rightarrow \infty$ this solution reduces to the one obtained in [30] up to the convention adopted there, where the fermionic four point function in the massive free fermion theory gauged by Chern-Simons interaction was computed. This is consistent with the fact that the Schwinger-Dyson equation (3.2) with (3.3) boils down to that of the Chern-Simons fermion vector model.

Secondly, by sending $\lambda, \frac{y_{2}^{2}}{\lambda}, \frac{y_{6}}{\lambda} \rightarrow 0$ with $\frac{2 \pi \lambda}{y_{4}}=g_{4}^{F}$ fixed this solution reduces to the four point function of Gross-Neveu model computed in [37]. Indeed in this limit all the components of $F_{\bar{\mu} \bar{\nu}}$ vanish except $F_{I I}$, which is given by

$$
\begin{aligned}
F_{I I} & =\frac{1}{N} \frac{g_{4}^{F}}{1+g_{4}^{F}\left(-\frac{c_{F}}{2 \pi}-\left(q_{3}^{2}+4 c_{F}^{2}\right) \frac{-\tan ^{-1} \frac{2 c_{F}}{q_{3}}+\tan ^{-1} \frac{2 \infty}{q_{3}}}{4 \pi q_{3}}\right)} \\
& =\frac{4 \pi}{N} \frac{q_{3}}{-\left(q_{3}^{2}+4 c_{F}^{2}\right) \tan ^{-1}\left(\frac{q_{3}}{2 c_{F}}\right)}
\end{aligned}
$$

where in the second equation we used $c_{F}=\frac{2 \pi}{g_{4}^{F}}$ under the limit and the formula $\tan ^{-1}(x)+$ $\tan ^{-1}\left(\frac{1}{x}\right)=\tan ^{-1}(\infty)$. Making (3.51) Lorentz covariant by exchanging $q_{3} \rightarrow \sqrt{\left(q_{\mu}\right)^{2}}$ gives (9) in [37] up to an overall numerical factor. 


\section{S-matrix and duality}

In this section we compute the two body scattering matrix of fermions in the general Chern-Simons fermion vector model in the 't Hooft large $N$ limit. For this we apply the method the S-matrix developed in [30], in which the fermionic S-matrix in the regular Chern-Simons fermion vector model was computed.

In the current system there are two kinds of scattering processes to be studied, that is particle-particle and particle-antiparticle scattering. ${ }^{2}$ We focus on the particle-antiparticle scattering in this paper since the particle-particle S-matrix is to be obtained by analytic continuation from the particle-antiparticle S-matrix as shown in [30]. Asymptotic states for this process are constructed in appendix B. We consider a particle-antiparticle scattering process such that

$$
\left|\vec{p}_{3},-, k ; \vec{p}_{2},+, j\right\rangle \rightarrow\left|-\vec{p}_{4},-, l ;-\vec{p}_{1},+, i\right\rangle .
$$

This means that the $k$-the particle with momentum $\vec{p}_{3}$ and the $j$-th antiparticle with momentum $\overrightarrow{p_{2}}$ come from the past infinity and go into the $l$-the particle with momentum $-\vec{p}_{4}$ and the $i$-th antiparticle with momentum $-\vec{p}_{1}$ at the future infinity. By taking into account momentum conservation, the S-matrix of this process can be written as

$$
\begin{aligned}
& \left\langle-\vec{p}_{4},-, l ;-\vec{p}_{1},+, i|\hat{S}| \vec{p}_{3},-, k ; \vec{p}_{2},+, j\right\rangle \\
& \quad=S\left(-\vec{p}_{4},-, l ;-\vec{p}_{1},+, i \mid \vec{p}_{3},-, k ; \vec{p}_{2},+, j\right)(2 \pi)^{3} \delta^{3}\left(p_{1}+p_{2}+p_{3}+p_{4}\right) .
\end{aligned}
$$

Furthermore the S-matrix can be decomposed in terms of the gauge indices as [30]

$$
\begin{aligned}
& S\left(-\vec{p}_{4},-, l ;-\vec{p}_{1},+, i \mid \vec{p}_{3},-, k ; \vec{p}_{2},+, j\right) \\
& =\left(\delta_{k}^{l} \delta_{i}^{j}-\frac{1}{N} \delta_{i}^{l} \delta_{k}^{j}\right) S_{T}\left(-\vec{p}_{4},-;-\vec{p}_{1},+\mid \vec{p}_{3},-; \vec{p}_{2},+\right)+\frac{1}{N} \delta_{k}^{j} \delta_{i}^{l} S_{S}\left(-\vec{p}_{4},-;-\vec{p}_{1},+\mid \vec{p}_{3},-; \vec{p}_{2},+\right) .
\end{aligned}
$$

We call the coefficients $S_{T}\left(-\vec{p}_{4},-;-\vec{p}_{1},+\mid \vec{p}_{3},-; \vec{p}_{2},+\right), S_{S}\left(-\vec{p}_{4},-;-\vec{p}_{1},+\mid \vec{p}_{3},-; \vec{p}_{2},+\right) \mathrm{T}-$ channel (adjoint channel) S-matrix, S-channel (singlet channel) one, respectively. Similarly the transition matrix defined by $\hat{S}=\hat{1}+i \hat{T}$ is decomposed as

$$
\begin{aligned}
& T\left(-\vec{p}_{4},-, l ;-\vec{p}_{1},+, i \mid \vec{p}_{3},-, k ; \vec{p}_{2},+, j\right) \\
& =\left(\delta_{k}^{l} \delta_{i}^{j}-\frac{1}{N} \delta_{i}^{l} \delta_{k}^{j}\right) T_{T}\left(-\vec{p}_{4},-;-\vec{p}_{1},+\mid \vec{p}_{3},-; \vec{p}_{2},+\right)+\frac{1}{N} \delta_{k}^{j} \delta_{i}^{l} T_{S}\left(-\vec{p}_{4},-;-\vec{p}_{1},+\mid \vec{p}_{3},-; \vec{p}_{2},+\right) .
\end{aligned}
$$

Then S-matrix for each channel satisfies

$$
\begin{aligned}
& S_{T}\left(-\vec{p}_{4},-;-\vec{p}_{1},+\mid \vec{p}_{3},-; \vec{p}_{2},+\right) \\
& =\frac{\left\langle-\vec{p}_{4},-;-\vec{p}_{1},+\mid \vec{p}_{3},-; \vec{p}_{2},+\right\rangle}{(2 \pi)^{3} \delta^{3}\left(p_{1}+p_{2}+p_{3}+p_{4}\right)}+i T_{T}\left(-\vec{p}_{4},-;-\vec{p}_{1},+\mid \vec{p}_{3},-; \vec{p}_{2},+\right), \\
& S_{S}\left(-\vec{p}_{4},-;-\vec{p}_{1},+\mid \vec{p}_{3},-; \vec{p}_{2},+\right) \\
& =\frac{\left\langle-\vec{p}_{4},-;-\vec{p}_{1},+\mid \vec{p}_{3},-; \vec{p}_{2},+\right\rangle}{(2 \pi)^{3} \delta^{3}\left(p_{1}+p_{2}+p_{3}+p_{4}\right)}+i T_{S}\left(-\vec{p}_{4},-;-\vec{p}_{1},+\mid \vec{p}_{3},-; \vec{p}_{2},+\right),
\end{aligned}
$$

\footnotetext{
${ }^{2}$ Antiparticle-antiparticle S-matrix can be obtained by charge conjugation of particle-particle one.
} 
where

$$
\left\langle-\vec{p}_{4},-;-\vec{p}_{1},+\mid \vec{p}_{3},-; \vec{p}_{2},+\right\rangle=(2 \pi)^{2} 2 E_{\vec{p}_{3}} \delta^{2}\left(\vec{p}_{3}+\vec{p}_{4}\right)(2 \pi)^{2} 2 E_{\vec{p}_{2}} \delta^{2}\left(\vec{p}_{2}+\vec{p}_{1}\right) .
$$

By definition the T-matrix in the T-channel is of order $1 / N$, while that in $\mathrm{S}$-channel is of order one.

Let us determine the unitarity condition for each channel. Let us start the unitarity condition with respect to the S-matrix

$$
\hat{S}^{\dagger} \hat{S}=1
$$

In terms of the transition matrix, this is written as

$$
-i\left(\hat{T}-\hat{T}^{\dagger}\right)=\hat{T}^{\dagger} \hat{T}
$$

Let us sandwich both sides inside the two particle states so that

$$
\frac{\left\langle-\vec{p}_{4},-, l ;-\vec{p}_{1},+, i\left|-i\left(\hat{T}-\hat{T}^{\dagger}\right)\right| \vec{p}_{3},-, k ; \vec{p}_{2},+, j\right\rangle}{(2 \pi)^{3} \delta^{3}\left(p_{1}+p_{2}+p_{3}+p_{4}\right)}=\frac{\left\langle-\vec{p}_{4},-, l ;-\vec{p}_{1},+, i\left|\hat{T}^{\dagger} \hat{T}\right| \vec{p}_{3},-, k ; \vec{p}_{2},+, j\right\rangle}{(2 \pi)^{3} \delta^{3}\left(p_{1}+p_{2}+p_{3}+p_{4}\right)} .
$$

By using (4.4) the left-hand side is computed as

$$
\begin{aligned}
\text { l.h.s. }=- & i\left\{\left(\delta_{k}^{l} \delta_{i}^{j}-\frac{1}{N} \delta_{i}^{l} \delta_{k}^{j}\right)\left(T_{T}\left(-\vec{p}_{4},-;-\vec{p}_{1},+\mid \vec{p}_{3},-; \vec{p}_{2},+\right)-T_{T}\left(\vec{p}_{3},-; \vec{p}_{2},+\mid-\vec{p}_{4},-;-\vec{p}_{1},+\right)^{*}\right)\right. \\
& \left.+\frac{1}{N} \delta_{k}^{j} \delta_{i}^{l}\left(T_{S}\left(-\vec{p}_{4},-;-\vec{p}_{1},+\mid \vec{p}_{3},-; \vec{p}_{2},+\right)-T_{S}\left(\vec{p}_{3},-; \vec{p}_{2},+\mid-\vec{p}_{4},-;-\vec{p}_{1},+\right)^{*}\right)\right\} .
\end{aligned}
$$

In order to compute the right-hand side we insert the identity operator

$$
\begin{array}{r}
1=\sum_{k, j} \int \frac{d^{3} r_{1}}{(2 \pi)^{3}} \frac{d^{3} r_{2}}{(2 \pi)^{3}}(2 \pi) \delta\left(r_{1}^{2}+c_{F}^{2}\right) \theta\left(r_{1}^{0}\right)(2 \pi) \delta\left(r_{2}^{2}+c_{F}^{2}\right) \theta\left(r_{2}^{0}\right) \\
\times\left|\vec{r}_{1},-, k ; \vec{r}_{2},+, j\right\rangle\left\langle\vec{r}_{1},-, k ; \vec{r}_{2},+, j\right|+\cdots
\end{array}
$$

where the ellipsis contains other many-particle states with more than 2-particles, which is suppressed in the $1 / N$ expansion. Then the right-hand side is computed as

$$
\begin{aligned}
\text { r.h.s. }= & \int \frac{d^{3} r_{1}}{(2 \pi)^{3}} \frac{d^{3} r_{2}}{(2 \pi)^{3}}(2 \pi) \delta\left(r_{1}^{2}+c_{B}^{2}\right) \theta\left(r_{1}^{0}\right)(2 \pi) \delta\left(r_{2}^{2}+c_{B}^{2}\right) \theta\left(r_{2}^{0}\right) \\
& \times\left\{\left(\delta_{k}^{l} \delta_{i}^{j}-\frac{\delta_{i}^{l} \delta_{k}^{j}}{N}\right) T_{T}\left(-\vec{p}_{4},-;-\vec{p}_{1},+\mid \vec{r}_{1},-; \vec{r}_{2},+\right) T_{T}\left(\vec{p}_{3},-; \vec{p}_{2},+\mid \vec{r}_{1},-; \vec{r}_{2},+\right)^{*}\right. \\
& \left.+\frac{\delta_{k}^{j} \delta_{i}^{l}}{N} T_{S}\left(-\vec{p}_{4},-;-\vec{p}_{1},+\mid \vec{r}_{1},-; \vec{r}_{2},+\right) T_{S}\left(\vec{p}_{3},-; \vec{p}_{2},+\mid \vec{r}_{1},-; \vec{r}_{2},+\right)^{*}\right\} .
\end{aligned}
$$

Recalling the fact that the transition matrix in the T-channel is of order $1 / N$, the product of $T_{T}$ and $T_{T}^{*}$ is of order $1 / N^{2}$, which is subleading in the $1 / N$ expansion. Thus to the leading order in the large $N$ limit the unitarity condition is given by

$$
-i\left\{T_{T}\left(-\vec{p}_{4},-;-\vec{p}_{1},+\mid \vec{p}_{3},-; \vec{p}_{2},+\right)-T_{T}\left(\vec{p}_{3},-; \vec{p}_{2},+\mid-\vec{p}_{4},-;-\vec{p}_{1},+\right)^{*}\right\}=0
$$


for the T-channel, and

$$
\begin{aligned}
- & i\left\{T_{S}\left(-\vec{p}_{4},-;-\vec{p}_{1},+\mid \vec{p}_{3},-; \vec{p}_{2},+\right)-T_{S}\left(\vec{p}_{3},-; \vec{p}_{2},+\mid-\vec{p}_{4},-;-\vec{p}_{1},+\right)^{*}\right\} \\
= & \int \frac{d^{3} r_{1}}{(2 \pi)^{3}} \frac{d^{3} r_{2}}{(2 \pi)^{3}}(2 \pi) \delta\left(r_{1}^{2}+c_{F}^{2}\right) \theta\left(r_{1}^{0}\right)(2 \pi) \delta\left(r_{2}^{2}+c_{F}^{2}\right) \theta\left(r_{2}^{0}\right) \\
& \times T_{S}\left(-\vec{p}_{4},-;-\vec{p}_{1},+\mid \vec{r}_{1},-; \vec{r}_{2},+\right) T_{S}\left(\vec{p}_{3},-; \vec{p}_{2},+\mid \vec{r}_{1},-; \vec{r}_{2},+\right)^{*}
\end{aligned}
$$

for the S-channel.

\section{$4.1 \quad$ T-channel}

We determine the transition matrix in the T-channel, which can be done by applying the LSZ formula to the fermion four point vertex determined in the previous section. Since the exact four point vertex was determined in Euclidean space, we perform the Wick rotation such that

$$
x^{2}=i x^{0}, \quad q^{2}=i q^{0} .
$$

In the momentum assignment in the previous section the T-channel is given by

$$
-k^{0}>0, p^{0}>0,(k+q)^{0}<0,-(p+q)^{0}<0 .
$$

Then the LSZ formula tells us a contraction rule of the wave functions associated with the external legs to obtain the T-channel transition matrix as follows.

$$
\begin{aligned}
T_{T} & =T_{T}(-\vec{k}-\vec{q},-; \vec{p}+\vec{q},+\mid-\vec{k},-; \vec{p},+) \\
& =v^{\alpha}(-\vec{k}-\vec{q}) \bar{v}_{\beta}(-\vec{k}) F_{{ }^{\prime}{ }^{\prime}{ }^{\beta}}{ }_{\alpha}(\vec{p}, \vec{k}, \vec{p}+\vec{q}) u^{\beta^{\prime}}(\vec{p}) \bar{u}_{\alpha^{\prime}}(\vec{p}+\vec{q}) \\
& =\left[\bar{u}(\vec{p}+\vec{q}) \gamma^{\bar{\nu}} u(\vec{p})\right]\left[\bar{v}(-\vec{k}) \gamma^{\bar{\mu}} v(-\vec{k}-\vec{q})\right] F_{\bar{\nu} \bar{\mu}}(\vec{p}, \vec{k}, \vec{p}+\vec{q})
\end{aligned}
$$

where $u(\vec{p}), v(\vec{p})$ are given in (B.4), (B.5). By plugging $F_{\bar{\nu} \bar{\mu}}$ determined in the previous section into this we obtain

$$
\begin{aligned}
T_{T} & =-\frac{4 \pi q_{3}}{i k_{F}}\left(z-\frac{f(0)+\frac{\left(2 \operatorname{sgn}(\lambda) c_{F}+i q_{3}\right)\left(4 c_{F}(\lambda-\operatorname{sgn}(\lambda))-4 \widetilde{y}_{4}+2 i q_{3}\right)}{\left(2 \operatorname{sgn}(\lambda) F_{F}-i q_{3}\right)\left(4 c_{F}(\lambda-\operatorname{sgn}(\lambda))-4 \widetilde{y}_{4}-2 i q_{3}\right)} f(\infty)}{f(0)-\frac{\left(2 \operatorname{sgn}(\lambda) c_{F}+i q_{3}\right)\left(4 c_{F}(\lambda-\operatorname{sgn}(\lambda))-4 \widetilde{y}_{4}+2 i q_{3}\right)}{\left(2 \operatorname{sgn}(\lambda) c_{F}-i q_{3}\right)\left(4 c_{F}(\lambda-\operatorname{sgn}(\lambda))-4 \widetilde{y}_{4}-2 i q_{3}\right)} f(\infty)}\right) \\
& =-\frac{4 \pi q_{3}}{i k_{F}}\left(z+i \tan X_{F}\left(q_{3}\right)\right)
\end{aligned}
$$

where we set

$$
z=\frac{(p+k)_{-}}{(p-k)_{-}}
$$

with $p_{-}=\frac{p^{1}+p^{0}}{\sqrt{2}}$, and

$$
X_{F}\left(q_{3}\right)=(\lambda-\operatorname{sgn}(\lambda)) \tan ^{-1}\left(\frac{q_{3}}{2 c_{F}}\right)-\tan ^{-1}\left(\frac{2\left(\widetilde{y}_{4}-c_{F}(\lambda-\operatorname{sgn}(\lambda))\right)}{q_{3}}\right) .
$$

A few comments seem appropriate. Firstly, this satisfies the unitary equation (4.14). This is because the unitarity condition can be written as $T_{T}=\left.T_{T}^{*}\right|_{q_{3} \rightarrow-q_{3}}$, which can be easily seen from (4.19). 
Secondly, by performing the duality transformation found in [17], this transition matrix maps the one describing two body scattering of bosonic particles in general Chern-Simons scalar theory. To see this we recall the duality transformation in [17].

$$
k_{F}=-k_{B}, \quad \lambda=\lambda_{B}-\operatorname{sgn}\left(\lambda_{B}\right), \quad y_{6}=\frac{1-x_{6}}{4}, \quad y_{4}=b_{4}, \quad y_{2}^{2}=m_{B}^{2},
$$

which suggests that $c_{F}=c_{B}$. Plugging this duality relation into (4.19) we obtain

$$
T_{T}=\frac{4 \pi q_{3}}{i k_{B}}\left(z+i \tan X_{B}\left(q_{3}\right)\right)
$$

where

$$
X_{B}\left(q_{3}\right)=\lambda_{B} \tan ^{-1}\left(\frac{q_{3}}{2 c_{B}}\right)+\tan ^{-1}\left(\frac{-4 b_{4}+c_{B} \lambda_{B}\left(1+3 x_{6}\right)}{2 q_{3}}\right) .
$$

This precisely agrees with the transition matrix in the T-channel (adjoint channel) in the general Chern-Simons bosonic vector model, $T_{T}^{(B)}$, which is computed in appendix C by extending the result in [30] including the triple trace coupling. Thus the S-matrix of this system given by (4.5) maps to the one of the dual bosonic system.

Thirdly one can make this scattering amplitude Lorentz covariant by rewriting in terms of Mandelstam variables. Our definition of Mandelstam variables is ${ }^{3}$

$$
s=-q^{2}, \quad t=-(p-k)^{2}, \quad u=-(p+k+q)^{2},
$$

which satisfy $s+t+u=4 c_{F}^{2}$. In the frame (3.12), $s=-q_{3}^{2}<0$. Let us consider to rewrite the first term in (4.19), which comes from the one gluon exchange diagram. This part can be made Lorentz covariant as

$$
-\frac{4 \pi q_{3}}{i k_{F}} z=-\frac{4 \pi}{i k_{F}} \frac{\varepsilon^{\mu \nu \rho}(p+k)_{\mu} q_{\nu}(p-k)_{\rho}}{(p-k)^{2}}
$$

where $\varepsilon^{013}=1$. From a straightforward algebraic calculation we can show that

$$
\left(\varepsilon^{\mu \nu \rho}(p+k)_{\mu} q_{\nu}(p-k)_{\rho}\right)^{2}=s t u .
$$

which leads to

$$
\varepsilon^{\mu \nu \rho}(p+k)_{\mu} q_{\nu}(p-k)_{\rho}=\sigma \sqrt{s t u} .
$$

where we set $\sigma=\operatorname{sgn}\left(\varepsilon^{\mu \nu \rho} k_{\mu} q_{\nu} p_{\rho}\right)$. Therefore we find

$$
-\frac{4 \pi q_{3}}{i k_{F}} z=-\frac{4 \pi}{i k_{F}} \frac{\sigma \sqrt{s t u}}{-t}=\frac{4 \pi}{k_{F}} \sigma \sqrt{\frac{s u}{-t}}
$$

where we used the fact that $t>0$ in the T-channel. Substituting these into (4.19) we obtain the Lorentz covariant form of the T-channel scattering amplitude as

$$
T_{T}=\frac{4 \pi}{k_{F}}\left(\sigma \sqrt{\frac{s u}{-t}}-\sqrt{-s} \tan X_{F}(\sqrt{-s})\right) .
$$

\footnotetext{
${ }^{3}$ The definition of Mandelstam variables in this paper is different from that in [30]. The $s, t, u$ variables there are defined in each channel so that $s$ always becomes positive as adopted in a standard textbook [38], while here they are defined globally so that $s, t, u$ become positive in S,T,U-channel, respectively.
} 


\subsection{S-channel}

Finally we determine the transition matrix in the S-channel. For this let us first compute the transition matrix in the S-channel in the same fashion as done in the T-channel. For the S-channel we perform a Wick rotation from Euclidean space such that

$$
x^{3}=i x^{0}, \quad q^{3}=i q^{0} .
$$

Then the frame (3.12) coincides with the center of mass frame for two body scattering. In the momentum assignment in the previous section S-channel is given by

$$
-(p+q)^{0}>0, p^{0}>0,(k+q)^{0}<0,-k^{0}<0 .
$$

In particular $q^{0}<0$. From the LSZ formula the S-channel transition matrix is obtained by contracting the four point vertex with the wave functions associated with the external legs in such a way that

$$
\begin{aligned}
T_{S} & =T_{S}(-\vec{k}-\vec{q},-; \vec{k},+\mid-\vec{p}-\vec{q},-; \vec{p},+) \\
& =-v^{\alpha}(-k-q) \bar{u}_{\beta}\left(k_{s}\right) F^{\alpha^{\prime}}{ }_{\beta^{\prime}}{ }^{\beta}{ }_{\alpha}(p, k, p+q) u^{\beta^{\prime}}(p) \bar{v}_{\alpha^{\prime}}(-p-q) \\
& =-\left[\bar{v}(-p-q) \gamma^{\bar{\nu}} u(p)\right]\left[\bar{u}\left(k_{s}\right) \gamma^{\bar{\mu}} v(-k-q)\right] F_{\bar{\nu} \bar{\mu}}(p, k, p+q)
\end{aligned}
$$

where $u(\vec{p}), v(\vec{p})$ are given in (B.4), (B.5) with double Wick rotation so that firstly $q^{0} \rightarrow$ $-i q^{2}$, subsequently $q^{3} \rightarrow i q^{0}$. Note that the minus sign appears when the fermionic fields associated the four point vertex contract with the external states. Plugging $F_{\bar{\nu} \bar{\mu}}$ determined in the previous section into this gives

$$
T_{S}=\frac{4 \pi q^{0}}{k_{F}}\left(z+\tanh X_{F}^{\prime}\left(-q^{0}\right)\right)
$$

where $z$ is given by $(4.20)$ with $p_{-}=\frac{p^{1}-i p^{2}}{\sqrt{2}}$ and

$$
X_{F}^{\prime}\left(-q^{0}\right)=(\lambda-\operatorname{sgn}(\lambda)) \tanh ^{-1}\left(\frac{-q^{0}}{2 c_{F}}\right)+\tanh ^{-1}\left(\frac{2\left(\widetilde{y}_{4}-c_{F}(\lambda-\operatorname{sgn}(\lambda))\right)}{-q^{0}}\right) .
$$

This result matches the one obtained by performing the double Wick rotation against the T-channel transition matrix given by (4.19). Performing the duality transformation we obtain

$$
T_{S}=-T_{S}^{(B)}
$$

where $T_{S}^{(B)}$ is given by (C.17).

A main problem of the S-channel transition matrix determined in this way is that it does not satisfy the unitarity condition [30]. In fact, the same problem of non-unitarity in the S-matrix also exists in the original Aharonov-Bohm scattering [39], which is to be obtained by taking the non-relativistic limit of the S-matrix in the current set up. The problem of non-unitarity in Aharonov-Bohm scattering was resolved by Ruijsenaars in [33], who pointed out that the delta-function type singular contribution in the forward scattering was missed in the original Aharonov-Bohm scattering and it becomes unitary by taking 
into account the singular contribution. The reason why this phenomenon happens may be that in the set up of Aharonov-Bohm scattering, where electrons are sent to screen in the presence of solenoid at the center, the contribution to the wave function of one path of electron through the left side of the solenoid and that of the right side are equal only in the forward scattering, whose coherent superposition is singular.

The method to cure the non-unitary problem in the relativistic situation developed in [30] is also to take into account the singular contribution in the forward scattering by the ansatz of the Schwinger-Dyson equation of the scattering amplitude such that

$$
T_{S}^{\mathrm{conj}}=T_{1}(\sqrt{s})+z T_{2}(\sqrt{s})+T_{3}(\sqrt{s}) 2 \pi \delta(\theta)
$$

where $\sqrt{s}=-q^{0}$ and $\theta$ is the scattering angle of an out-particle to the line formed by two in-particles in the center of mass frame (3.12). By using $\theta$ the on-shell momenta of in and out particles can be parametrized as

$$
p^{\mu}=(E, p, 0), \quad k^{\mu}=(E, p \cos \theta, p \sin \theta), \quad E=\sqrt{p^{2}+c_{F}^{2}}=\frac{\sqrt{s}}{2}
$$

which leads to $z=-i \cot \frac{\theta}{2}$. $T_{3}$ describes the singular contribution in the forward scattering. Then the unitarity condition (4.15) boils down to

$$
\begin{aligned}
-i T_{1}+i T_{1}^{*} & =\frac{1}{4 \sqrt{s}}\left(\left|T_{1}\right|^{2}+T_{1}^{*} T_{3}+T_{1} T_{3}^{*}-\left|T_{2}\right|^{2}\right), \\
-i T_{2}+i T_{2}^{*} & =\frac{1}{4 \sqrt{s}}\left(T_{2}^{*} T_{3}+T_{2} T_{3}^{*}\right), \\
-i T_{3}+i T_{3}^{*} & =\frac{1}{4 \sqrt{s}}\left(\left|T_{2}\right|^{2}+\left|T_{3}\right|^{2}\right),
\end{aligned}
$$

where we used $\sqrt{s}>2 c_{F}$ in the S-channel scattering.

A solution physically preferable may be as follows. Whether the situation is relativistic or not does not matter in the argument for the singular term to be generated, so it may be safe to assume that the singular part is the same as in the non-relativistic case.

$$
T_{3}=4 i \sqrt{s}(1-\cos \pi \lambda) \text {. }
$$

Then (4.40) is met if $T_{2}$ is real. In addition by using (4.41) $T_{2}$ is determined as

$$
T_{2}=-4 \sqrt{s} \sin \pi \lambda
$$

Finally (4.39) is met if $T_{3}$ is given by

$$
T_{1}=-4 \sqrt{s} \sin (\pi \lambda) \tanh \left(X_{F}^{\prime}(\sqrt{s})\right) .
$$

The solution found in this way possesses several desired properties as argued in [30] as follows.

(i) Unitarity is guaranteed by construction. 
(ii) The S-matrix constructed this way enjoys the bosonization duality. To see this let us compute the S-channel S-matrix by plugging the above solution into (4.6). The first term in (4.6) by using (4.38) can be computed as

$$
\frac{\left\langle-\vec{r}_{4},-;-\vec{r}_{1},+\mid \vec{r}_{3},-; \vec{r}_{2},+\right\rangle}{(2 \pi)^{3} \delta^{3}\left(r_{1}+r_{2}+r_{3}+r_{4}\right)}=8 \pi \sqrt{s} \delta(\theta) .
$$

Then the result is

$$
S_{S}^{\mathrm{conj}}=i k_{F} \frac{\sin (\pi \lambda)}{\pi} T_{S}+8 \pi \sqrt{s} \cos (\pi \lambda) \delta(\theta)
$$

where $T_{S}$ is given by (4.34). ${ }^{4}$ Due to (4.36) the conjectural S-matrix in the S-channel transforms under the duality transformation as

$$
S_{S}^{\mathrm{conj}}=-i k_{B} \frac{\sin \left(\pi \lambda_{B}\right)}{\pi} T_{S}^{(B)}-8 \pi \sqrt{s} \cos \left(\pi \lambda_{B}\right) \delta(\theta)=-S_{S}^{(B), \text { conj }},
$$

where in the second equation we used (C.19). This shows that the physical scattering amplitudes coincide in the dual theories.

(iii) Upon analytically continuing the S-channel S-matrix to off-shell region $\sqrt{s}<2 c_{F}$, a pole arises in a certain coupling region [40]. The bound state energy is determined by the following equation

$$
y=\lambda+\frac{2(\mathfrak{s}+\operatorname{sgn}(\lambda)) \mathfrak{s}}{(\mathfrak{s}-\operatorname{sgn}(\lambda)) e^{2 \lambda \tanh ^{-1}(\mathfrak{s})}+\mathfrak{s}+\operatorname{sgn}(\lambda)}-\mathfrak{s}-\operatorname{sgn}(\lambda)
$$

where we set $\mathfrak{s}=\frac{\sqrt{s}}{2 c_{F}}, y=\frac{\widetilde{y}_{4}}{c_{F}}$. In other words, a particle-antiparticle bound state shows up when this equation has a solution. The region for a solution to exist is $1<\frac{y}{\lambda}<\frac{2-|\lambda|}{1-|\lambda|}{ }^{5}$ In particular in the limit $y_{4}, y_{2}^{2} \rightarrow \infty$ with $\lambda, y_{6}, \frac{y_{2}^{2}}{y_{4}}$ fixed, in which this system reduces to the regular Chern-Simons fermionic vector model [17], the bound state disappears, which is consistent with the result in [41]. In addition the bound states in the dual theories map to each other under the duality transformation [31].

(iv) The non-relativistic limit reduces the solution to the Aharonov-Bohm-Ruijsenaars scattering amplitude. To see this let us take the non-relativistic limit $\sqrt{s} \rightarrow 2 c_{B}$ for the S-channel transition matrix with general coupling constants. The result is

$$
T_{S}^{\mathrm{conj}} \rightarrow 8 c_{F}\left(-\sin |\pi \lambda|-\sin |\pi \lambda| i \cot \frac{\theta^{\prime}}{2}+i(1-\cos \pi \lambda) 2 \pi \delta\left(\theta^{\prime}\right)\right)
$$

where we redefine the scattering angle so that $\theta^{\prime}=-\operatorname{sgn}(\lambda) \theta$. In order to compare the scattering function in quantum mechanics, we scale the result by $\frac{1}{c_{F} \sqrt{p}}$ (see [30]),

\footnotetext{
${ }^{4}$ The delta function $\delta(\theta)$ can be written in terms of the Mandelstam variables as $\delta(\theta)=\frac{1}{2} \delta\left(\sqrt{\frac{t}{u}}\right)$.

${ }^{5}$ This region may be related to that where the theory possesses the reflection positivity. In fact, the reflection positivity of the two point correlator of the auxiliary field with fermion loops excluded implies that $k_{F} \widetilde{y}_{4}>0$, which is compatible with the region for the pole to arise.
} 
which correctly reproduces the Aharonov-Bohm-Ruijsenaars scattering amplitude up to an overall numerical factor.

Connection to the self-adjoint extension of Aharonov-Bohm scattering [42] was found in [40]. This was achieved by taking the non-relativistic limit and simultaneously sending the couplings to the lower threshold for the bound state to exist, where the mass of the bound state approaches $2 c_{F}$. By denoting deviations from the bounds by

$$
\sqrt{s}=2 c_{F}+\epsilon_{1} \frac{p^{2}}{c_{F}}, \quad \widetilde{y}_{4}=c_{F}\left(\lambda+\epsilon_{2}\right)
$$

the limit is given by

$$
\epsilon_{1}, \epsilon_{2} \rightarrow 0, \quad \frac{\epsilon_{2}}{\epsilon_{1}^{\lambda_{B}}}: \text { fixed }
$$

in the assumption that $\lambda<0$ or $\lambda_{B}>0$. Under this limit the improved transition matrix in the bosonic side reduces to

$$
T_{S}^{\mathrm{conj},(\mathrm{B})} \rightarrow-8 c_{B}\left[\sin \pi \lambda_{B}\left(\frac{\left(\frac{2 c_{B}}{p}\right)^{2 \lambda_{B}} \frac{\epsilon_{2}}{2 \epsilon_{1}^{\lambda_{B}}} e^{i \pi \lambda_{B}}-1}{\left(\frac{2 c_{B}}{p}\right)^{2 \lambda_{B}} \frac{\epsilon_{2}}{2 \epsilon_{1}^{\lambda_{B}}} e^{i \pi \lambda_{B}}+1}-i \cot \frac{\theta}{2}\right)+i\left(\cos \pi \lambda_{B}-1\right) 2 \pi \delta(\theta)\right] .
$$

On the other hand, the Aharonov-Bohm scattering with a general self-adjoint boundary condition is given by [42]

$f(\theta)=\frac{-i e^{\frac{-i \pi}{4}}}{\sqrt{2 \pi p}}\left[\sin \pi \alpha\left(\frac{\frac{\Gamma(1+\alpha)}{w \Gamma(1-\alpha)}\left(\frac{2}{R p}\right)^{2 \alpha} e^{i \pi \alpha}-1}{\frac{\Gamma(1+\alpha)}{w \Gamma(1-\alpha)}\left(\frac{2}{R p}\right)^{2 \alpha} e^{i \pi \alpha}+1}-i \cot \frac{\theta}{2}\right)+2 \pi i(\cos \alpha \pi-1) \delta(\theta)\right]$

where $p$ is the initial momentum, $\alpha$ is the background magnetic field normalized so as to be in the range from 0 to 1 , and $R$ is the effective radius of the system. They agree with each other up to an overall factor mentioned above under the identification such that

$$
\frac{\epsilon_{2}}{\epsilon_{1}^{\lambda_{B}}}=\frac{2 \Gamma(1+\alpha)}{w \Gamma(1-\alpha)}, \quad c_{B}=\frac{1}{R}, \quad \lambda_{B}=\alpha
$$

Finally this result suggests that the usual crossing symmetry given in standard textbooks of quantum field theory needs to be modified when the scattering amplitude contains a singular contribution. In the current system upon performing analytic continuation from T-channel to S-channel the amplitude picks up the overall factor $N \times \frac{\sin \pi \lambda}{\pi \lambda}$. The origin of this factor was discussed in relation to Wilson lines formed in the trajectory of the charged particles [30], though such a novel aspect is not confirmed from explicit calculation yet. We leave it to future work.

\section{Discussion}

We have computed the four point function of the fermionic field exactly in the general $\mathrm{U}(N)$ Chern-Simons fermionic vector model in the 't Hooft large $N$ limit. Applying the 
LSZ formula to this result with a suitable Wick rotation, we have calculated scattering amplitudes in the T-channel and the S-channel taking special care for the S-channel so as to include the singular contribution in the forward scattering to achieve unitarity. We have shown that the scattering amplitudes determined in this way enjoy the bosonization duality proposed in [17] as well as a novel crossing relation proposed in [30]. We have also shown that in the non-relativistic limit in a general coupling region the S-matrix reduces to the Aharonov-Bohm-Ruijsenaars scattering amplitude, and in a special coupling region of the threshold for the bound state to exist the S-matrix reduces to the self adjoint extension of Aharonov-Bohm scattering [42] as claimed in [40].

It turned out that the conjectural S-matrix in tje S-channel in the general ChernSimons fermionic vector model develops a pole in a certain coupling region, which is a signal for a particle-antiparticle bound state to exist. Analysis of a bound state in the regular Chern-Simons fermionic vector model was done from the Bethe-Salpeter equation with the result of no bound state in that system [41], which is consistent with the result in this paper. It would be interesting to derive the Bethe-Salpeter equation in the current system and determine the bound state energy, which is expected to match the one determined in this paper.

As discussed in [17] the Chern-Simons vector models in the current paper are expected to be dual to a higher-spin gravity theory with parity violated by the boundary condition in $\mathrm{AdS}_{4}[1,2]$. In the gravity side three point correlation functions were computed by taking advantage of infinitely many higher spin symmetries [43-45]. (See also $[8,10]$.) It is interesting to compute the counterpart in the gravity side for the scattering amplitude computed in the paper.

It would be interesting to recompute the scattering amplitude including chemical potential as done in [32], where the S-matrix in Chern-Simons theory with dense fermionic matter was computed and was applied to determining Landau parameters to see the consistency of a microscopic calculation and a thermodynamic macroscopic one. It would be intriguing to determine Landau parameters in the current setup and investigate its behavior in the RG flow and the physical implication thereof.

It would be also interesting to compute the scattering amplitude in higher supersymmetric case such as ABJ model by taking the vector model large $N$ limit. Some perturbative calculation of two body scattering in $\mathrm{ABJ}(\mathrm{M})$ theory was done in [46-52]. In the results there the singular term in forward scattering does not show up, while the result in the $\mathcal{N}=1,2$ Chern-Simons vector model exhibits the singular contribution for the unitarity [31]. This issue may be cleared up by carrying out the large $N$ calculation developed in the analysis of Chern-Simons vector models explicitly.

We hope to make progress in these issues in the near future.

\section{Acknowledgments}

The author would like to thank S. Jain, M. Mandlik and S. Minwalla for collaboration and valuable discussions. He would also like to thank O. Baer for reading the manuscript. 
This work was supported by the MEXT-Supported Program for the Strategic Research Foundation at Private Universities "Topological Science" (Grant No. S1511006).

\section{A Regularization}

In this appendix we detail the regularization for the divergence we encountered in section 3 following [18]. The divergent integrals are contained in (3.34), (3.38), which are of a form such that

$$
I=\int_{\mathfrak{h}}^{\infty} \frac{d h}{(2 \pi)} \frac{A_{i}(h)\left(2 i \Sigma_{I}+q_{3}\right)+2 B_{i}(h)\left(h^{2}-\Sigma_{I}^{2}\right)}{4 h^{2}+q_{3}^{2}}
$$

where $\mathfrak{h}=\sqrt{\mathfrak{p}_{s}^{2}+c_{F}^{2}}$ and we denote $A_{i}\left(k_{s}^{\prime}, k_{s}\right)=A_{i}(h), B_{i}\left(k_{s}^{\prime}, k_{s}\right)=B_{i}(h)$ with $h=h_{k^{\prime}}=$ $\sqrt{\left(k_{s}^{\prime}\right)^{2}+c_{F}^{2}}$. Using (3.28) and (3.30) one can show that the asymptotic behavior of the numerator in the integrand around $h \sim \infty$ is

$$
A_{i}(h)\left(2 i \Sigma_{I}+q_{3}\right)+2 B_{i}(h)\left(h^{2}-\Sigma_{I}^{2}\right)=2 B_{i}(\infty) h^{2}+\mathcal{O}\left(h^{0}\right) .
$$

Using this we can divide the above divergent integral into two parts in a way that

$$
I=\int_{\mathfrak{h}}^{\infty} \frac{d h}{(2 \pi)}\left[\frac{A_{i}(h)\left(2 i \Sigma_{I}+q_{3}\right)+2 B_{i}(h)\left(h^{2}-\Sigma_{I}^{2}\right)-2 B_{i}(\infty) h^{2}}{4 h^{2}+q_{3}^{2}}+\frac{2 B_{i}(\infty) h^{2}}{4 h^{2}+q_{3}^{2}}\right] .
$$

The first term, which we denote by $I_{1}$, is convergent and computed as

$$
\begin{aligned}
I_{1}= & \lim _{\Lambda \rightarrow \infty} \int_{\mathfrak{h}}^{\Lambda} \frac{d h}{(2 \pi)}\left[\frac{A_{i}(h)\left(2 i \Sigma_{I}+q_{3}\right)+2 B_{i}(h)\left(h^{2}-\Sigma_{I}^{2}\right)-2 B_{i}(\Lambda) h^{2}}{4 h^{2}+q_{3}^{2}}\right] \\
= & \frac{1}{16 \pi \lambda}\left\{\alpha_{i} \lambda\left(\pi \operatorname{sgn}\left(q_{3}\right)-2 \tan ^{-1}\left(\frac{2 \mathfrak{h}}{q_{3}}\right)\right)+2 \beta_{i} f\left(\mathfrak{p}_{s}\right)\left(2 c_{F}(\lambda-\operatorname{sgn}(\lambda))-2 \mathfrak{h} \lambda+i q_{3}\right)\right. \\
& \left.+\beta_{i} f(\infty)\left(-4 c_{F}(\lambda-\operatorname{sgn}(\lambda))+4 \mathfrak{h} \lambda-2 \lambda q_{3} \tan ^{-1}\left(\frac{2 \mathfrak{h}}{q_{3}}\right)+\pi \lambda q_{3} \operatorname{sgn}\left(q_{3}\right)-2 i q_{3}\right)\right\} .
\end{aligned}
$$

The second term, which we denote by $I_{2}$, is a divergent term, which we can easily regularize by the dimensional regularization prescribed in [18]. For this purpose we rewrite the integral as the original three-dimensional integral form.

$$
I_{2}=2 B_{i}(\infty) \int \frac{d^{3} k^{\prime}}{(2 \pi)^{3}} \frac{\left(k_{s}^{\prime}\right)^{2}+c_{F}^{2}}{\left(k^{\prime 2}+c_{F}^{2}\right)\left(\left(k^{\prime}+q\right)^{2}+c_{F}^{2}\right)} \frac{k_{-}^{\prime}}{\left(k^{\prime}-\mathfrak{p}\right)_{-}} .
$$

Then we can apply the dimensional regularization prescribed in [18] to this integration.

$$
I_{2}=2 B_{i}(\infty) \int \frac{d^{2} k^{\prime} d^{1-\epsilon} \mathbf{k}_{3}}{(2 \pi)^{3}} \frac{h^{2}}{\left(\left(\mathbf{k}_{3}\right)^{2}+h^{2}\right)\left(\left(\mathbf{k}_{3}+\mathbf{q}_{3}\right)^{2}+h^{2}\right)} \frac{k_{-}^{\prime}}{\left(k^{\prime}-\mathfrak{p}\right)_{-}} .
$$

This can be computed in a standard method by employing Feynman parameters. The result is

$$
I_{2}=2 B_{i}(\infty) \times \frac{-1}{8 \pi}\left(\mathfrak{h}-\frac{q_{3}}{2} \tan ^{-1} \frac{2 \mathfrak{h}}{q_{3}}+\frac{\pi\left|q_{3}\right|}{4}\right) .
$$

Finally we obtain the regulated integral as

$$
\begin{aligned}
I & =I_{1}+I_{2} \\
& =\frac{-2 \alpha_{i} \mathfrak{h} \lambda+\beta_{i} q_{3} f\left(\mathfrak{p}_{s}\right)\left(2 c_{F}(\lambda-\operatorname{sgn}(\lambda))-2 \mathfrak{h} \lambda+i q_{3}\right)+\beta_{i} q_{3} f(\infty)\left(-2 c_{F} \lambda+2 c_{F}-i q_{3}\right)}{8 \pi \lambda q_{3}} .
\end{aligned}
$$




\section{B Construction of asymptotic states}

In this appendix we construct asymptotic particle states which are necessary to define the S-matrix. For this purpose we quantize the fermionic field of the system (2.1) in the canonical formulation. The Dirac equation of the system (2.1) is

$$
\left(\gamma_{\mu} \partial^{\mu}+\Sigma_{F}\right) \psi_{x}^{m}=0
$$

where $m$ is the fundamental index of the $\mathrm{U}(N)$ gauge group and $\Sigma_{F}$ is given by (2.4). To find a complete set of the solutions we do Fourier transformation:

$$
\left(i \gamma_{\mu} p^{\mu}+\Sigma_{F}(p)\right) \psi_{p}^{m}=0 .
$$

A non-trivial solution of the Dirac equation exists only when the momentum is on massshell:

$$
p^{0}= \pm E_{\vec{p}}, \quad E_{\vec{p}}:=\sqrt{\vec{p}^{2}+c_{F}^{2}} .
$$

Then a positive-energy solution is found to be

$$
u(\vec{p})=\left(\frac{-i p_{3}+\Sigma_{I}(p)}{\sqrt{p^{1}+p^{0}}},-i \sqrt{p^{1}+p^{0}}\right),
$$

with $p^{0}=E_{\vec{p}}$, and negative-energy one is

$$
v(-\vec{p})=\left(\frac{i p_{3}-\Sigma_{I}(p)}{\sqrt{p^{1}+p^{0}}}, i \sqrt{p^{1}+p^{0}}\right),
$$

with $p^{0}=-E_{\vec{p}}$. These are normalized in such a way that

$$
\bar{u}(\vec{p}) u(\vec{p})=2 \Sigma_{I}(p), \quad \bar{v}(-\vec{p}) v(-\vec{p})=-2 \Sigma_{I}(p) .
$$

Then we expand the fermionic field in terms of this complete set. The result is

$$
\psi_{x}^{m}=\left.\int \frac{d^{2} p}{(2 \pi)^{2}} \frac{1}{\sqrt{2 E_{\vec{p}}}}\left(a_{\vec{p}}^{m} u(\vec{p}) e^{i x p}+\left(b_{m, \vec{p}}\right)^{\dagger} v(\vec{p}) e^{-i x p}\right)\right|_{p^{0}=E_{\vec{p}}}
$$

where $a_{\vec{p}}, b_{\vec{p}}$ are expansion coefficients of the field.

Following the canonical formalism we introduce the canonical commutation relation.

$$
\left\{a_{\vec{p}}^{m},\left(a_{\vec{q}}^{n}\right)^{\dagger}\right\}=(2 \pi)^{2} \delta_{n}^{m} \delta^{2}(\vec{p}-\vec{q}), \quad\left\{b_{m, \vec{p}},\left(b_{n, \vec{q}}\right)^{\dagger}\right\}=(2 \pi)^{2} \delta_{m}^{n} \delta^{2}(\vec{p}-\vec{q}) .
$$

Then $\left(a_{\vec{p}}^{m}\right)^{\dagger},\left(b_{m, \vec{p}}\right)^{\dagger}$ are one-particle creation operators with the positive, negative charges, respectively. We call the particle with positive charge antiparticle. By using these creation operators we can define in-state and out-state of one particle by

$$
\begin{array}{rlrl}
|\vec{p},+, m\rangle & =\sqrt{2 E_{\vec{p}}}\left(a_{\vec{p}}^{m}\right)^{\dagger}|0\rangle, & |\vec{p},-, m\rangle=\sqrt{2 E_{\vec{p}}}\left(b_{m, \vec{p}}\right)^{\dagger}|0\rangle, \\
\langle\vec{p},+, m|=\sqrt{2 E_{\vec{p}}}\left(0 \mid a_{\vec{p}}^{m},\right. & \langle\vec{p},-, m|=\sqrt{2 E_{\vec{p}}}\left(0 \mid b_{m, \vec{p}},\right.
\end{array}
$$


where $|0\rangle$ is the vacuum state. Here the normalization is determined so that the inner product of these state becomes Lorentz invariant:

$$
\langle\vec{p},+, m \mid \vec{q},+, n\rangle=\langle\vec{p},-, n \mid \vec{q},-, m\rangle=2 E_{\vec{p}}(2 \pi)^{2} \delta_{n}^{m} \delta^{2}(\vec{q}-\vec{p}) .
$$

In the main text we study two particle scattering, where two particle states are defined by

$$
\begin{aligned}
|\vec{q},-, n ; \vec{p},+, m\rangle & =\sqrt{2 E_{\vec{q}}}\left(b_{n, \vec{q}}\right)^{\dagger} \sqrt{2 E_{\vec{p}}}\left(a_{\vec{p}}^{m}\right)^{\dagger}|0\rangle, \\
\langle\vec{q},-, n ; \vec{p},+, m| & =\sqrt{2 E_{\vec{p}}} \sqrt{2 E_{\vec{q}}}\langle 0| a_{\vec{p}}^{m} b_{n, \vec{q}} .
\end{aligned}
$$

\section{S-matrix in general Chern-Simons bosonic vector theory}

In this appendix we give a brief derivation of two body scattering amplitude in general Chern-Simons vector model in the 't Hooft large $N$ limit. This can be done by applying the method developed in [30] to the current case, in which the triple trace coupling is included. Lagrangian of general Chern-Simons bosonic vector model is [15, 17]

$$
\begin{aligned}
\mathcal{L}_{B}= & i \varepsilon^{\mu \nu \rho} \frac{k_{B}}{4 \pi} \operatorname{Tr}\left(A_{\mu} \partial_{\nu} A_{\rho}-\frac{2 i}{3} A_{\mu} A_{\nu} A_{\rho}\right)+D_{\mu} \bar{\phi} D^{\mu} \phi \\
& +m_{B}^{2} \bar{\phi} \phi+\frac{4 \pi b_{4}}{k_{B}}(\bar{\phi} \phi)^{2}+\frac{(2 \pi)^{2} x_{6}}{\left(k_{B}\right)^{2}}(\bar{\phi} \phi)^{3} .
\end{aligned}
$$

The exact propagator of the scalar field is determined as [15]

$$
\left\langle\phi^{m}(p) \bar{\phi}_{n}\left(-p^{\prime}\right)\right\rangle=\delta_{n}^{m}(2 \pi)^{3} \delta^{3}\left(-p^{\prime}+p\right) \alpha_{B}(p), \quad \alpha_{B}(p)=\frac{1}{p^{2}+c_{B}^{2}}
$$

where $c_{B}^{2}$ is the physical mass of the scalar field determined by the following gap equation

$$
c_{B}^{2}=\lambda_{B}^{2}\left(1+3 x_{6}\right) \frac{c_{B}^{2}}{4}-2 \lambda_{B} b_{4} c_{B}+m_{B}^{2} .
$$

As in the fermionic case in order to compute the four point correlator we determine the four point vertex given by

$$
-\frac{1}{2} \int \frac{d^{3} p}{(2 \pi)^{3}} \frac{d^{3} k}{(2 \pi)^{3}} \frac{d^{3} q}{(2 \pi)^{3}} \bar{\phi}_{m}(-p-q) \phi^{m}(p) B(p, k ; q) \bar{\phi}_{n}(-k) \phi^{n}(k+q) .
$$

As the fermionic case, the bootstrap diagram for this four point vertex is precisely the same as the ladder diagram in the 't Hooft large $N$ limit, thus the Schwinger-Dyson equation is

$$
B(p, k ; q)=B^{0}(p, k ; q)+N \int \frac{d^{3} k^{\prime}}{(2 \pi)^{3}} B^{0}\left(p, k^{\prime} ; q\right) \alpha_{B}\left(q+k^{\prime}\right) \alpha_{B}\left(k^{\prime}\right) B\left(k^{\prime}, k ; q\right) .
$$

Here $B^{0}$ represents the contribution of the ladder bar, which is diagrammatically given by figure 4 in [30] with the contribution of the triple trace vertex given by

$$
-\left(\frac{2 \pi}{k_{B}}\right)^{2} \times 3 ! \times N \int \frac{d^{3} r}{(2 \pi)^{3}} \alpha_{B}(r)=6 x_{6}\left(\frac{2 \pi}{k_{B}}\right)^{2} N \frac{c_{B}}{4 \pi} .
$$


Thus we obtain

$$
B^{0}(p, k ; q)=\frac{4 \pi}{i k_{B}} q_{3} z+\frac{\mathfrak{b}_{4}}{k_{B}}
$$

where $\mathfrak{b}_{4}$ is a constant given by

$$
\mathfrak{b}_{4}=2 \pi \lambda_{B} c_{B}-8 \pi b_{4}+6 \pi x_{6} \lambda_{B} c_{B}
$$

The bootstrap equation (C.5) can be solved exactly in the frame (3.12). In order to solve (C.5) we set the ansatz [6]

$$
B(p, k ; q)=B_{1}\left(p_{s}, k_{s}\right)+q_{3} z B_{2}\left(p_{s}, k_{s}\right),
$$

where $z$ is defined by (4.20). Plugging this into (C.5) and performing $k_{3}$-integral and angular integral for $k_{-}$we find

$$
\begin{aligned}
B_{2}\left(p_{s}, k_{s}\right)= & \frac{4 \pi}{i k_{B}}+N \int_{h_{p}}^{h_{k}} \frac{d h}{(2 \pi)} \frac{1}{\left(4 h^{2}+q_{3}^{2}\right)} \frac{-8 \pi q_{3}}{i k_{B}} B_{2}, \\
B_{1}\left(p_{s}, k_{s}\right)= & \frac{\mathfrak{b}_{4}}{k_{B}}+N \int_{h_{0}}^{h_{p}} \frac{d h}{(2 \pi)} \frac{1}{\left(4 h^{2}+q_{3}^{2}\right)} \frac{8 \pi q_{3}}{i k_{B}} B_{1}+N \int_{h_{0}}^{h_{k}} \frac{d h}{(2 \pi)} \frac{1}{\left(4 h^{2}+q_{3}^{2}\right)} \frac{-2 \mathfrak{b}_{4} q_{3}}{k_{B}} B_{2} \\
& +N \int_{h_{0}}^{\infty} \frac{d h}{(2 \pi)} \frac{1}{\left(4 h^{2}+q_{3}^{2}\right)}\left(\frac{-4 \pi q_{3}}{i k_{B}}+\frac{\mathfrak{b}_{4}}{k_{B}}\right)\left(B_{1}+q_{3} B_{2}\right)
\end{aligned}
$$

where in the right-hand side we set $B_{i}=B_{i}\left(k_{s}^{\prime}, k_{s}\right)$ with $h=h_{k^{\prime}}$. A solution of these integral equations is

$$
\begin{aligned}
& B_{2}\left(p_{s}, k_{s}\right)=\frac{4 \pi}{i k_{B}} \frac{f_{B}\left(p_{s}\right)}{f_{B}\left(k_{s}\right)} \\
& B_{1}\left(p_{s}, k_{s}\right)=\frac{4 \pi q_{3}}{i k_{B}}\left[-\frac{\left(\mathfrak{b}_{4}+i 4 \pi q_{3}\right) f_{B}(\infty)+\left(\mathfrak{b}_{4}-i 4 \pi q_{3}\right) f_{B}(0)}{\left(\mathfrak{b}_{4}+i 4 \pi q_{3}\right) f_{B}(\infty)-\left(\mathfrak{b}_{4}-i 4 \pi q_{3}\right) f_{B}(0)}\right] \frac{f_{B}\left(p_{s}\right)}{f_{B}\left(k_{s}\right)},
\end{aligned}
$$

where $f_{B}\left(p_{s}\right)$ is defined by

$$
f_{B}\left(p_{s}\right):=\exp \left[-2 i \lambda_{B} \arctan \frac{2 \sqrt{p_{s}^{2}+c_{B}^{2}}}{q_{3}}\right] .
$$

Therefore

$$
B(p, k ; q)=\frac{4 \pi q_{3}}{i k_{B}}\left(z+i \tan X_{B}\left(q_{3}\right)\right) \frac{f_{B}\left(p_{s}\right)}{f_{B}\left(k_{s}\right)}
$$

where $X_{B}\left(q_{3}\right)$ is given by $(4.24)$.

Then the procedure to compute scattering amplitude is completely parallel to the fermionic case described in the main text. Therefore we do not repeat the description for the bosonic case, and we present only results with the subscript $(B)$ for the corresponding quantities. The T-channel transition matrix is

$$
T_{T}^{(B)}=\frac{4 \pi q_{3}}{i k_{B}}\left(z+i \tan X_{B}\left(q_{3}\right)\right)=-\frac{4 \pi}{k_{B}}\left(\sigma \sqrt{\frac{s u}{-t}}-\sqrt{-s} \tan X_{B}(\sqrt{-s})\right),
$$


and the naive S-channel transition matrix obtained by double Wick rotation is

$$
T_{S}^{(B)}=\frac{4 \pi q^{0}}{k_{B}}\left(z+\tanh X_{B}^{\prime}\left(-q^{0}\right)\right)=-\frac{4 \pi}{k_{B}}\left(\sigma \sqrt{\frac{s u}{-t}}-\sqrt{s} \tanh X_{B}^{\prime}(\sqrt{s})\right),
$$

where $s, t, u$ variables are defined by (4.25) and

$$
X_{B}^{\prime}(\sqrt{s})=\lambda_{B} \tanh ^{-1} \frac{\sqrt{s}}{2 c_{B}}+\tanh ^{-1}\left(\frac{-4 b_{4}+c_{B} \lambda_{B}\left(1+3 x_{6}\right)}{2 \sqrt{s}}\right) .
$$

By making the S-channel S-matrix unitary as done in [30] we obtain

$$
S_{S}^{(B), \text { conj }}=i k_{B} \frac{\sin \left(\pi \lambda_{B}\right)}{\pi} T_{S}^{(B)}+8 \pi \sqrt{s} \cos \left(\pi \lambda_{B}\right) \delta(\theta) .
$$

Open Access. This article is distributed under the terms of the Creative Commons Attribution License (CC-BY 4.0), which permits any use, distribution and reproduction in any medium, provided the original author(s) and source are credited.

\section{References}

[1] S. Giombi, S. Minwalla, S. Prakash, S.P. Trivedi, S.R. Wadia and X. Yin, Chern-Simons theory with vector fermion matter, Eur. Phys. J. C 72 (2012) 2112 [arXiv:1110.4386] [INSPIRE].

[2] O. Aharony, G. Gur-Ari and R. Yacoby, $D=3$ bosonic vector models coupled to Chern-Simons gauge theories, JHEP 03 (2012) 037 [arXiv:1110.4382] [INSPIRE].

[3] J.M. Maldacena, The large- $N$ limit of superconformal field theories and supergravity, Int. J. Theor. Phys. 38 (1999) 1113 [hep-th/9711200] [INSPIRE].

[4] I.R. Klebanov and A.M. Polyakov, AdS dual of the critical $O(N)$ vector model, Phys. Lett. B 550 (2002) 213 [hep-th/0210114] [INSPIRE].

[5] C.-M. Chang, S. Minwalla, T. Sharma and X. Yin, ABJ triality: from higher spin fields to strings, J. Phys. A 46 (2013) 214009 [arXiv:1207.4485] [inSPIRE].

[6] O. Aharony, G. Gur-Ari and R. Yacoby, Correlation functions of large- $N$ Chern-Simons-matter theories and bosonization in three dimensions, JHEP 12 (2012) 028 [arXiv: 1207.4593] [INSPIRE].

[7] G. Gur-Ari and R. Yacoby, Correlators of large- $N$ fermionic Chern-Simons vector models, JHEP 02 (2013) 150 [arXiv:1211.1866] [INSPIRE].

[8] S. Giombi, S. Prakash and X. Yin, A note on CFT correlators in three dimensions, JHEP 07 (2013) 105 [arXiv: 1104.4317] [INSPIRE].

[9] J. Maldacena and A. Zhiboedov, Constraining conformal field theories with a higher spin symmetry, J. Phys. A 46 (2013) 214011 [arXiv:1112.1016] [InSPIRE].

[10] J. Maldacena and A. Zhiboedov, Constraining conformal field theories with a slightly broken higher spin symmetry, Class. Quant. Grav. 30 (2013) 104003 [arXiv: 1204.3882] [INSPIRE].

[11] A.A. Nizami, T. Sharma and V. Umesh, Superspace formulation and correlation functions of $3 d$ superconformal field theories, JHEP 07 (2014) 022 [arXiv:1308.4778] [INSPIRE]. 
[12] A. Bedhotiya and S. Prakash, A test of bosonization at the level of four-point functions in Chern-Simons vector models, JHEP 12 (2015) 032 [arXiv:1506.05412] [INSPIRE].

[13] O. Aharony, S. Giombi, G. Gur-Ari, J. Maldacena and R. Yacoby, The thermal free energy in large-N Chern-Simons-matter theories, JHEP 03 (2013) 121 [arXiv:1211.4843] [INSPIRE].

[14] S. Jain, S. Minwalla, T. Sharma, T. Takimi, S.R. Wadia and S. Yokoyama, Phases of large- $N$ vector Chern-Simons theories on $S^{2} \times S^{1}$, JHEP 09 (2013) 009 [arXiv: 1301.6169] [INSPIRE].

[15] S. Jain, S. Minwalla and S. Yokoyama, Chern Simons duality with a fundamental boson and fermion, JHEP 11 (2013) 037 [arXiv:1305.7235] [INSPIRE].

[16] S. Yokoyama, A note on large- $N$ thermal free energy in supersymmetric Chern-Simons vector models, JHEP 01 (2014) 148 [arXiv: 1310.0902] [INSPIRE].

[17] S. Minwalla and S. Yokoyama, Chern Simons bosonization along RG flows, JHEP 02 (2016) 103 [arXiv:1507.04546] [INSPIRE].

[18] S. Jain, S.P. Trivedi, S.R. Wadia and S. Yokoyama, Supersymmetric Chern-Simons theories with vector matter, JHEP 10 (2012) 194 [arXiv:1207.4750] [INSPIRE].

[19] S. Yokoyama, Chern-Simons-fermion vector model with chemical potential, JHEP 01 (2013) 052 [arXiv: 1210.4109] [INSPIRE].

[20] T. Takimi, Duality and higher temperature phases of large- $N$ Chern-Simons matter theories on $S^{2} \times S^{1}$, JHEP 07 (2013) 177 [arXiv: 1304.3725] [INSPIRE].

[21] M. Moshe and J. Zinn-Justin, 3D field theories with Chern-Simons term for large- $N$ in the Weyl gauge, JHEP 01 (2015) 054 [arXiv: 1410.0558] [INSPIRE].

[22] G. Gur-Ari and R. Yacoby, Three dimensional bosonization from supersymmetry, JHEP 11 (2015) 013 [arXiv: 1507.04378] [INSPIRE].

[23] A. Giveon and D. Kutasov, Seiberg duality in Chern-Simons theory, Nucl. Phys. B 812 (2009) 1 [arXiv: 0808.0360] [INSPIRE].

[24] F. Benini, C. Closset and S. Cremonesi, Comments on 3d Seiberg-like dualities, JHEP 10 (2011) 075 [arXiv: 1108.5373] [InSPIRE].

[25] S. Banerjee, S. Hellerman, J. Maltz and S.H. Shenker, Light states in Chern-Simons theory coupled to fundamental matter, JHEP 03 (2013) 097 [arXiv: 1207.4195] [INSPIRE].

[26] Y. Frishman and J. Sonnenschein, Breaking conformal invariance - large- $N$ Chern-Simons theory coupled to massive fundamental fermions, JHEP 12 (2013) 091 [arXiv:1306.6465] [INSPIRE].

[27] W.A. Bardeen and M. Moshe, Spontaneous breaking of scale invariance in a $D=3 U(N)$ model with Chern-Simons gauge fields, JHEP 06 (2014) 113 [arXiv:1402.4196] [INSPIRE].

[28] V. Gurucharan and S. Prakash, Anomalous dimensions in non-supersymmetric bifundamental Chern-Simons theories, JHEP 09 (2014) 009 [arXiv:1404.7849] [INSPIRE].

[29] D. Radičević, Disorder operators in Chern-Simons-fermion theories, JHEP 03 (2016) 131 [arXiv: 1511.01902] [INSPIRE].

[30] S. Jain, M. Mandlik, S. Minwalla, T. Takimi, S.R. Wadia and S. Yokoyama, Unitarity, crossing symmetry and duality of the S-matrix in large- $N$ Chern-Simons theories with fundamental matter, JHEP 04 (2015) 129 [arXiv:1404.6373] [INSPIRE]. 
[31] K. Inbasekar, S. Jain, S. Mazumdar, S. Minwalla, V. Umesh and S. Yokoyama, Unitarity, crossing symmetry and duality in the scattering of $N=1$ SUSY matter Chern-Simons theories, JHEP 10 (2015) 176 [arXiv: 1505.06571] [INSPIRE].

[32] M. Geracie, M. Goykhman and D.T. Son, Dense Chern-Simons matter with fermions at large-N, JHEP 04 (2016) 103 [arXiv: 1511.04772] [INSPIRE].

[33] S.N.M. Ruijsenaars, The Aharonov-Bohm effect and scattering theory, Annals Phys. 146 (1983) 1 [INSPIRE].

[34] S.C. Zhang, T.H. Hansson and S. Kivelson, An effective field theory model for the fractional quantum Hall effect, Phys. Rev. Lett. 62 (1988) 82 [INSPIRE].

[35] E. Fradkin and A. Lopez, Fractional quantum Hall effect and Chern-Simons gauge theories, Phys. Rev. B 44 (1991) 5246 [InSPIRE].

[36] D.J. Gross and A. Neveu, Dynamical symmetry breaking in asymptotically free field theories, Phys. Rev. D 10 (1974) 3235 [inSPIRE].

[37] B. Rosenstein, B.J. Warr and S.H. Park, The four Fermi theory is renormalizable in (2+1)-dimensions, Phys. Rev. Lett. 62 (1989) 1433 [InSPIRE].

[38] M.E. Peskin and D.V. Schroeder, An introduction to quantum field theory, Addison-Wesley, Reading U.S.A. (1995) [INSPIRE].

[39] Y. Aharonov and D. Bohm, Significance of electromagnetic potentials in the quantum theory, Phys. Rev. 115 (1959) 485 [INSPIRE].

[40] Y. Dandekar, M. Mandlik and S. Minwalla, Poles in the S-matrix of relativistic Chern-Simons matter theories from quantum mechanics, JHEP 04 (2015) 102 [arXiv: 1407.1322] [INSPIRE].

[41] Y. Frishman and J. Sonnenschein, Large- $N$ Chern-Simons with massive fundamental fermions - a model with no bound states, JHEP 12 (2014) 165 [arXiv:1409.6083] [INSPIRE].

[42] G. Amelino-Camelia and D. Bak, Schrödinger selfadjoint extension and quantum field theory, Phys. Lett. B 343 (1995) 231 [hep-th/9406213] [INSPIRE].

[43] S. Giombi and X. Yin, Higher spin gauge theory and holography: the three-point functions, JHEP 09 (2010) 115 [arXiv: 0912.3462] [INSPIRE].

[44] S. Giombi and X. Yin, Higher spins in AdS and twistorial holography, JHEP 04 (2011) 086 [arXiv: 1004.3736] [INSPIRE].

[45] S. Giombi and X. Yin, On higher spin gauge theory and the critical $O(N)$ model, Phys. Rev. D 85 (2012) 086005 [arXiv:1105.4011] [INSPIRE].

[46] A. Agarwal, N. Beisert and T. McLoughlin, Scattering in mass-deformed $N \geq 4$ Chern-Simons models, JHEP 06 (2009) 045 [arXiv:0812.3367] [INSPIRE].

[47] M.S. Bianchi, M. Leoni, A. Mauri, S. Penati and A. Santambrogio, Scattering in ABJ theories, JHEP 12 (2011) 073 [arXiv:1110.0738] [INSPIRE].

[48] W.-M. Chen and Y.-T. Huang, Dualities for loop amplitudes of $N=6$ Chern-Simons matter theory, JHEP 11 (2011) 057 [arXiv:1107.2710] [INSPIRE].

[49] M.S. Bianchi, M. Leoni, A. Mauri, S. Penati and A. Santambrogio, Scattering amplitudes/Wilson loop duality in ABJM theory, JHEP 01 (2012) 056 [arXiv:1107.3139] [INSPIRE]. 
[50] M.S. Bianchi, M. Leoni, A. Mauri, S. Penati and A. Santambrogio, One loop amplitudes in ABJM, JHEP 07 (2012) 029 [arXiv: 1204.4407] [INSPIRE].

[51] T. Bargheer, N. Beisert, F. Loebbert and T. McLoughlin, Conformal anomaly for amplitudes in $N=6$ superconformal Chern-Simons theory, J. Phys. A 45 (2012) 475402 [arXiv: 1204.4406] [INSPIRE].

[52] M.S. Bianchi and M. Leoni, On the ABJM four-point amplitude at three loops and BDS exponentiation, JHEP 11 (2014) 077 [arXiv:1403.3398] [INSPIRE]. 